Tropical Journal of Pharmaceutical Research March 2019; 18 (3): 647-667

ISSN: $1596-5996$ (print); 1596-9827 (electronic)

(C) Pharmacotherapy Group, Faculty of Pharmacy, University of Benin, Benin City, 300001 Nigeria.

\title{
External application of Ruyi Jinhuang powder for phlebitis: A systematic review and meta-analysis
}

\author{
Qian Yang ${ }^{1}$, Chunyan Yang ${ }^{2}$, Yongqiong Deng ${ }^{3}$, Qunhua Ma ${ }^{4 *}$ \\ ${ }^{1}$ Nursing School, Southwest Medical University, No. 3, 319 Section of ZhongShan Road, Jiangyang District, ${ }^{2}$ Department of \\ Dermatology, The Affiliated Traditional Chinese Medicine Hospital of Southwest Medical University, No. 16, Chunhui Road, \\ Longma Tan District, ${ }^{3}$ Department of Dermatology, The Affiliated Hospital of Southwest Medical University, No. 25 Taiping \\ Street, Jiangyang District, Luzhou 646000, ${ }^{4}$ Sichuan Academy of Medical Sciences \& Sichuan Provincial People's Hospital \\ MICU, Chengdu, Sichuan 610072, China
}

*For correspondence: Email: qunhua_ma@163.com; Tel/Fax: +86 28-87393999

Sent for review: 21 June 2017

Revised accepted: 17 February 2019

\begin{abstract}
Purpose: To systematically review the effectiveness of the external application, Ruyi Jinhuang powder, on phlebitis

Methods: Relevant literature was retrieved from Medline, Embase, China National Knowledge Infrastructure database (CNKI), Cochrane Central Register, Chongqing Vip, Wanfang Data and SinoMed using the search terms "Ruyi Jinhuang San", "Ruyi Jinhuang powder", "Ruyi Jinhuang cream", "Agreeable golden powder", "satisfactory golden powder", "Jinhuang cream", "phlebitis", "prevention and (or) treatment", "randomized controlled trials" and "RCRs". Two researchers independently arranged and analyzed the data.

Results: Significant differences were observed in the total effectiveness rate of Ruyi Jinhuang powder [relative risk $(R R)=1.27,95 \%$ confidence interval $(C l)=1.19$ to 1.36 , and $p<0.0001)$. Ruyi Jinhuang powder can reduce the incidence of phlebitis versus conventional therapy in preventing phlebitis $(R R=$ $0.32,95 \% \mathrm{Cl} 0.24$ to 0.42 , and $p<0.0001$ ). Shorten the average healing time [mean difference $(M D)=-$ $32.17,95 \% \mathrm{Cl}=-48.39$ to -15.94 , and $p=0.0001)$. Reduced pain relief time for phlebitis ( $M D=-3.29$, $95 \% \mathrm{Cl}-5.42$ to -1.16 , and $p=0.002)$. However, no statistical difference was observed with regard to the onset time of phlebitis ( $M D=-0.62,95 \% \mathrm{Cl}-1.76$ to 0.52 , and $p=0.29$ ).

Conclusion: Clinicians consider Ruyi Jinhuang powder a viable complementary and alternative medicine for phlebitis following the stronger evidence being offered.
\end{abstract}

Keywords: Ruyi Jinhuang powder; Prevention and treatment; Phlebitis; Meta-analysis

\begin{abstract}
This is an Open Access article that uses a funding model which does not charge readers or their institutions for access and distributed under the terms of the Creative Commons Attribution License (http://creativecommons.org/licenses/by/4.0) and the Budapest Open Access Initiative (http://www.budapestopenaccessinitiative.org/read), which permit unrestricted use, distribution, and reproduction in any medium, provided the original work is properly credited.

Tropical Journal of Pharmaceutical Research is indexed by Science Citation Index (SciSearch), Scopus, International Pharmaceutical Abstract, Chemical Abstracts, Embase, Index Copernicus, EBSCO, African Index Medicus, JournalSeek, Journal Citation Reports/Science Edition, Directory of Open Access Journals (DOAJ), African Journal Online, Bioline International, Open-J-Gate and Pharmacy Abstracts
\end{abstract}

\section{INTRODUCTION}

In clinical settings, over $80 \%$ of hospitalized inpatients are given intravenous therapy upon admission [1]. A majority of drugs are administered via intravenous injection to maintain highly efficient bioavailability. Approximately, 20 to $70 \%$ of drugs may provoke infusion-induced phlebitis, which is a common and painful complication of peripheral intravenous cannulation. Such complication significantly reduces the drug compliance of patients. $^{2}$ 
Patients with intravenous injection of antibiotics and antineoplastic drugs may develop a high risk of infusion - induced phlebitis. Peripherally inserted central catheters (PICC) - induced Mechanic phlebitis, indwelling needle-induced phlebitis; superficial thrombophlebitis, etc [2]. Phlebitis cause a cascade of unwelcome repercussions-significant swelling, erythema, pain, warmth, thickening and hardening of the injection site and fever [3,4]. Increased prevalence of thrombophlebitis may disrupt the follow-up and negatively impact the physical health of patients. More importantly, the incidence of bacterial phlebitis can compromise subsequent provoke the occurrence of blood infection [5].

Age, gender smoking habit, peripheral vascular diseases, repeated intravenous catheterization, location and duration of catheter indwelling, catheter length and materials, interval of replacement, infusion and filtration devices, type of infused medications that causes lack of sensation (peripheral neuropathy), high-pH drugs and solutions were potentially considered as the risk factors. Other medications and intravenous fluids (vancomycin and benzylpenicillin antibiotics, aminophylline, amiodarone hydrochloride and potassium chloride, aminophylline and amiodarone hydrochloride and $7.4 \%$ calcium glubionate, were identified with the strongest phlebitis potential [2-4].

Heparin is associated with the risk of bleeding from the operation site and thrombocytopenia. Corticosteroids are followed by an increased risk of infection through impaired defense system [9]. Local use of anti-inflammatory medications is employed to alleviate the pain of superficial thrombophlebitis, reduce the acute inflammation and discomforts. Nevertheless, whether these therapies can avoid relevant complications remains to be further validated. Thus, it is recommended to use simpler, more economical, and available methods. One of these methods appears to be the use of complementary and alternative medicine.

In China, TCM has been applied to treat human diseases for more than 2000 years. In the history of TCM, physicians have accumulated clinical experience in management of phlebitis [10]. As an integral part of TCM, external application of Ruyi Jinhuang Powder yields lower cost, less adverse events and higher efficacy compared with traditional treatment. Ruyi Jinhuang Powder could also be used on inflammatory external hemorrhoids, acute gouty arthritis anddon't increase adverse reactions [11]. Considerable number of studies demonstrated that Ruyi
Jinhuang powder had various pharmacological effects including anti-inflammatory, anti-bacterial, and analgesic effects [12]. Moreover, Ruyi Jinhuang powder could promote granulation, adsorb wound secretion and keep the wound relatively moist [13]. However, the evidence of effectiveness and safety of phlebitis has not been assessed systematically. In addition, substantial numbers of studies could potentially be missed if literature searches are restricted to English-only sources [10].

In previous meta-analyses, the use of Ruyi Jinhuang powder has been applied to treat phlebitis [8,9]. They concentrated on the use of Ruyi Jinhuang powder compared with magnesium sulfate alone. The two metaanalyses were performed in 2010 and 2012, respectively.

\section{METHODS}

\section{Data source search}

To identify relevant randomized clinical trials (RCTs), two reviewers (Qian Yang and Jian Zhang) performed systematical search from the Medline, Embase, China National Knowledge Infrastructure database (CNKI), Cochrane Central Register, Chongqing Vip, Wanfang Data and SinoMed using the search terms "Ruyi Jinhuang San", "Ruyi Jinhuang powder", "Ruyi Jinhuang cream", "Agreeable golden powder", "satisfactory golden powder", "Jinhuang cream", "phlebitis", "prevention and (or) treatment", "randomized controlled trials" and "RCRs." In this meta-analysis, the papers published until September 2016 were researched and included. The reference list of the chosen publications was searched to obtain more relevant articles. We did not limit the language and type of publications. Conference proceedings, abstract-only articles and graduation dissertation were all selected if they met our inclusion criteria (Figure 1).

\section{Study selection}

\section{Studies}

Random control trials (RCTs) were included. Non-RCTs, Quasi-RCTs, or randomized trials with false randomization method design, mixed intervention, duplicate publication of data, no prescribed duration of treatment, complex TCM formula combined with Ruyi Jinhuang powder, inappropriate clinical outcome assessment and no data for extraction were excluded. 


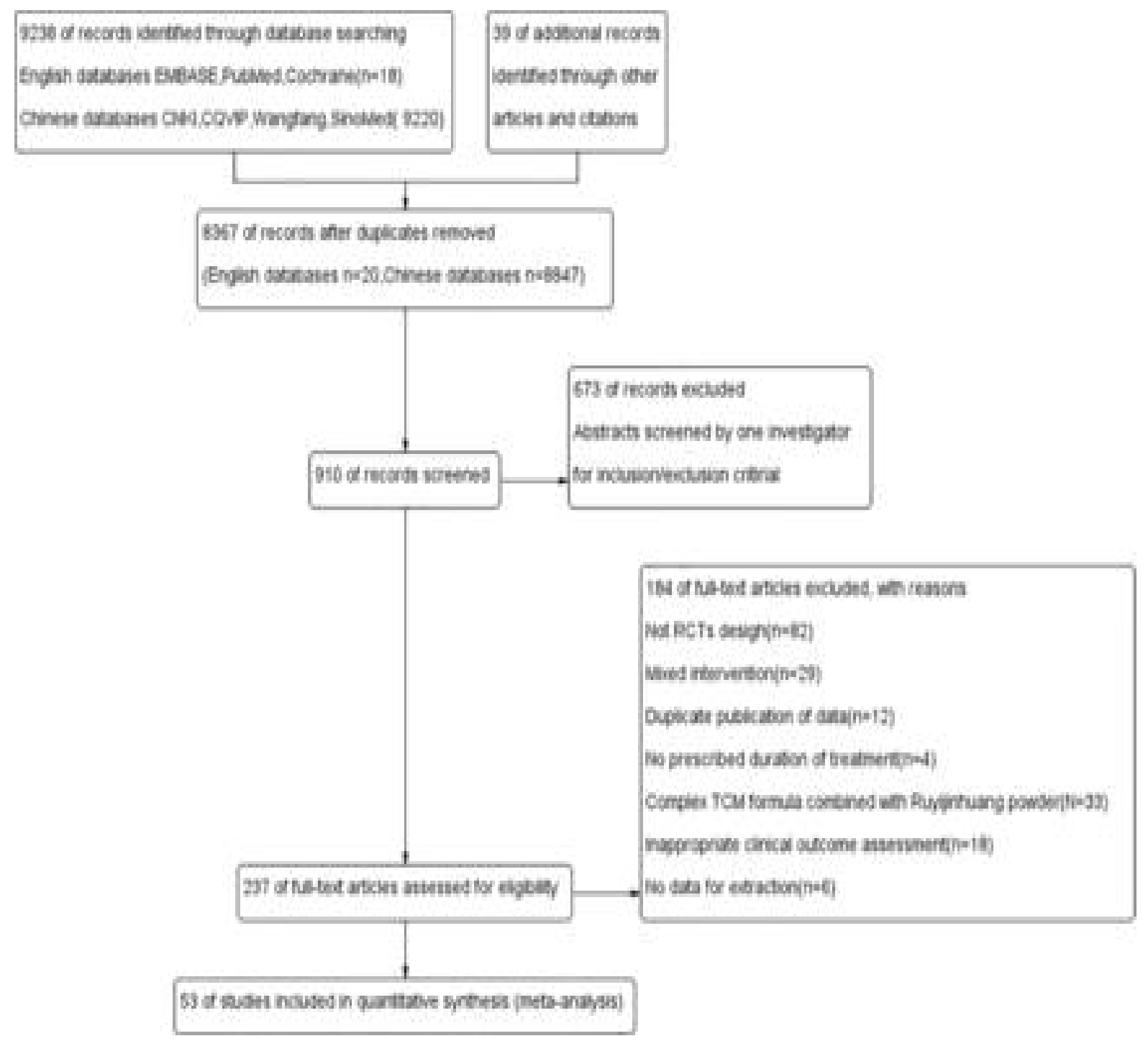

Figure 1: Summary of the literature identification and selection process. CNKI indicates the Chinese National Knowledge Infrastructure database; CQVIP, the Chinese Scientific Journals Full Text database; SinoMed, the Chinese Biomedical Literature Service System; RCT, randomized clinical trials.

\section{Participants}

Patients diagnosed with phlebitis based on any set of explicit criteria were included. No restrictive limitations were set on age, gender, nationality, or basic diseases, intravenous injection drugs, phlebitis classification. Retrieval results included 17 chemotherapeutic phlebitis [11-27], 10 PICC-induced phlebitis [28-37], 19 infusion phlebitis [38-56], 7 intravenous indwelling needle-induced phlebitis [57-63] (Table 1 to Table 12). All phlebitis classifications were evaluated based on the Infusion Nursing Standards of Practice proposed by the American Infusion Nurses Society in 2006 [64].

\section{Interventions}

The focused experimental groups received external application of Ruyi Jinhuang Powder. We did not set limitations on dosages, intervals, times and mixtures of it, or types of conventional therapy used. Our comparison of Ruyi Jinhuang powder and conventional therapy included Ruyi Jinhuang powder alone $[12,28,30,32,51]$ or Ruyi Jinhuang powder mixed with vinegar $[11,14-$ $17,38,48,52,54,59,60]$ mixed with honey $[1,19$ $21,23,25,27,29,34,37,39,41,45,46,50,57,58,63]$ mixed with ethano $[18,42,49,61]$ mixed with tea $[22,33,35,43,44,47]$ mixed with sesameoil $[24,31,40,62]$ mixed with water $[26,36]$ or mixed with glycerin $[53,55,56]$. The wound sites were dabbed dry with sterile gauze, or the wounds were not debrided but cleaned with normal saline gauze. If they were soiled, then the combined mixtures would be smeared onto the wounds with a sterile gloved finger. 


\section{Outcome measurement}

To more accurately assess the efficacy of Ruyi jinhuang powder, the primary outcomes as total effectiveness rate (TER) in treating phlebitis, incidence of phlebitis (IP) for preventing phlebitis applied with Ruyi Jinhuang powder were evaluated. The secondary outcomes included average healing time (AHT), pain relief time (PRT) and incidence time of phlebitis (ITP).

\section{Data extraction}

Two reviewers (Qian Yang and Jian Zhang) extracted data independently using a predetermined inclusion criteria. Disagreements were properly solved through mutual discussion or reaching a consensus by the third reviewer (Wen Xin Yang). The data extracted included the first author, year of publication, mean age, sample size, duration of trial period, basic diseases, intravenous infusion drugs, phlebitis classification, topical treatment of control group/experimental group. For studies lacking of sufficient information, the primary authors were contacted to obtain and validate the data. The use of modified JADAD scale evaluation, mainly includes 4 aspects as follow: (1) the generation of random sequence; (2) random hidden; (3) whether the use of blind method; (4) loss of access and withdrawal from the report. The highest score is 7 points, the lowest is divided into 0 points. At present, $1-3$ is considered as a low quality, and 4-7 is considered as a high quality (Table 1). Data extraction and quality evaluation process, if there are different views, the use of collective discussion method solved it.

\section{Risk of bias assessment}

The risk of bias in each study was assessed by two independent authors (Yong Qiong Deng and Xian Deng) using the Cochrane Risk of Bias tool [65] and disagreements were solved either by consensus or the third reviewer (Wen Xin Yang). Risk of bias and methodological quality of the selected papers are illustrated in Figure 2.

\section{RESULTS}

\section{Study selection}

The full texts of 237 relevant studies were reviewed from 9277 titles to validate the study eligibility.

Among 237 studies, 184 trials were excluded, including non-RCTs design $(n=82)$, mixed intervention $(n=29)$, duplicate publication of data $(n=12)$, no prescribed duration of treatment $(n=4)$, complex TCM formula combined with Ruyi Jinhuang powder $(n=33)$, inappropriate clinical outcome assessment $(n=18)$, no data for extraction $(n=6)$. Finally, 53 trials met the inclusion criteria and incorporated into the systematic review. All the selected studies were accomplished in China.

\section{Study characteristics}

All included 53 trials were published in Chinese language. In total, 4119 participants were enrolled in these trials $(n=2078$ in the experimental group and $n=2041$ in the control group). The sample sizes of these trials ranged from 40 to 240 (Table 1). The dose and frequency of the Ruyi Jinhuang powder differed in each trial. External application of $50 \%$ magnem sulphate is the most frequently used in 34 trials. Further therapeutic and preventive measures used in clinical trials.

\section{Risk of bias assessment}

The methodological quality of all selected trials was poor (Figure 2). All trials reported the randomization, but merely 12 explicitly described the randomization approach, 6 with random number table $[14,17,25,32,36,46], 3$ with random case sequence $[28,39,65]$, one the envelope method [30], one based on the admission date [58] and one with convenience sampling method [60], detailed information regarding random sequence generation was absent in the other trials.

Moreover, allocation concealment or blinding of participants and study personnel were described in only one of the trials [36], all of the relevant trials adequately addressed incomplete outcome data, selective reporting could not be judged in all the studies because of the insufficient information provided. We found no other biases in the studies due to low methodological quality. An unclear risk of bias was delivered to all selected studies.

\section{Primary outcomes}

\section{Incidence of phlebitis}

Fourteen RCTs containing 1418 patients illustrated the results, the experimental group included 714 trials, while the control group included 704 trials, the experimental and control groups received Ruyi jinhuang powder and conventional therapy, respectively. All subjects received fundamental interventions, the 14 independent trials showed heterogeneity in the consistency $\left(x^{2}=20.60, p=0.08, F^{2}=37 \%\right)$. 
Table 1: Basic characteristics of the included studies

\begin{tabular}{|c|c|c|c|c|c|c|c|c|}
\hline Study & $\begin{array}{l}\text { Sample size } \\
\text { E/C }\end{array}$ & $\begin{array}{l}\text { Sex or Age E/C } \\
\text { (Mean } \pm \text { SD) } \\
\text { years }\end{array}$ & $\begin{array}{l}\text { Duration } \\
E / C \text { (months) }\end{array}$ & $\begin{array}{l}\text { Phlebitis E/C } \\
\text { basic Diseases E/C } \\
\text { Drugs E/C } \\
\text { Phlebitis classification E/C }\end{array}$ & $\begin{array}{l}\text { Topical treatment of } \\
\text { control group }\end{array}$ & $\begin{array}{l}\text { External application } \\
\text { of experimental } \\
\text { group }\end{array}$ & $\begin{array}{l}\text { Main } \\
\text { outcomes }\end{array}$ & JADAD \\
\hline $\begin{array}{l}\text { Zhang and } \mathrm{Li} \\
2016 \text { [11] }\end{array}$ & $80(40 / 40)$ & $\begin{array}{l}\text { Sex:E:25/15 } \\
\text { C:23/17 } \\
\text { Age: } E: m=58 \\
C: M=53\end{array}$ & $\begin{array}{l}2012.12- \\
2013.12\end{array}$ & $\begin{array}{l}\text { Chemotherapy-induced phlebitis } \\
\text { Chemotherapeutic drugs: vinorelbine, } \\
\text { paclitaxel, fluorouracil, leurocristine, } \\
\text { cisplatin, carboplatin, } \\
\text { Cyclophosphamide, pharmorubicin) }\end{array}$ & $\begin{array}{l}\text { Give } 0.9 \% \text { normal saline } \\
\text { water or } 5 \% \text { glucose } 10-20 \\
\mathrm{~mL} \text { fast rapid intravenous } \\
\text { injection, and to dilute the } \\
\text { residual chemical therapy } \\
\text { drug content on blood vessel } \\
\text { walls, and from the reducing } \\
\text { of veins }\end{array}$ & $\begin{array}{l}\text { External application of } \\
\text { Ruyijinghuang Powder } \\
\text { combined with winegar } \\
\text { at } 1 \mathrm{~mm} \text { thickness for } \\
12 \text { hours, once a day } \\
\text { for } 5 \text { days }\end{array}$ & IP & 3 \\
\hline $\begin{array}{l}\text { Yang et al } \\
2013 \text { [12] }\end{array}$ & $60(30 / 30)$ & $\begin{array}{l}\text { Sex:All women } \\
\text { Age:E:28-53 } \\
M=45 \\
C: 27-55 M=46\end{array}$ & 2011.2-2012.9 & $\begin{array}{l}\text { Chemotherapy-induced phlebitis } \\
\text { Chemotherapeutic drugs: Fluorouracil } \\
\text { department of gynecology }\end{array}$ & 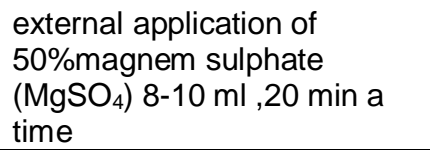 & $\begin{array}{l}\text { external application of } \\
\text { Ruyijinghuang Powder } \\
\text { at } 2 \mathrm{~mm} \text { thickness, } \\
\text { once a day }\end{array}$ & IP & 3 \\
\hline $\begin{array}{l}\text { Jiang } 2010 \\
{[13]}\end{array}$ & $50(25 / 25)$ & $\begin{array}{l}\text { Sex:All women } \\
\text { Age: } 35-60 \\
M=45\end{array}$ & $\begin{array}{l}2008.1- \\
2009.2\end{array}$ & $\begin{array}{l}\text { Chemotherapy-induced phlebitis } \\
\text { Diseased:radical mastectomy } \\
\text { Chemotherapeutic drugs } \\
\text { Cyclophosphamide, pharmorubicin, } 5 \\
\text { fluorouracil }\end{array}$ & $\begin{array}{l}\text { Conventional care not take } \\
\text { preventive measures }\end{array}$ & $\begin{array}{l}\text { External application of } \\
\text { Ruyijinhuang powder } \\
15 \mathrm{~g} \text { mixed with honey } \\
\text { at } 2 \mathrm{~mm} \text { thickness for } \\
8 \text { hours, twice a day }\end{array}$ & IP & 3 \\
\hline Li 2012 [14] & $(85 / 83)$ & $\begin{array}{l}\text { Sex:55/45 } \\
\text { Age:30-68 }\end{array}$ & $\begin{array}{l}2010.3- \\
2011.3\end{array}$ & $\begin{array}{l}\text { Chemotherapy-induced phlebitis non- } \\
\text { small cell lung cancer, breast cancer, } \\
\text { acute nonlymphocytic leukemia } \\
\text { Blistering chemotherapy drugs } \\
\text { (vinorelbine, Doxorubicin) }\end{array}$ & $\begin{array}{l}\text { Dexamethasone sodium5 } \\
\text { mg, sodium aescinate } 5 \\
\text { mg, add into } 10 \mathrm{ml} \text { sodium } \\
\text { chloride solution, } \\
\text { intravenous push for } \\
\text { several times }\end{array}$ & $\begin{array}{l}\text { external application } \\
\text { of Ruyijinghuang } \\
\text { Powder combined } \\
\text { with winegar at } 5 \mathrm{~mm} \\
\text { thickniss for } 24 \\
\text { hours }\end{array}$ & IP & 4 \\
\hline $\begin{array}{l}\text { Cheng } 2012 \\
{[15]}\end{array}$ & $120(60 / 60)$ & $\begin{array}{l}\text { Sex:68/52 } \\
\text { Age:28-76 }\end{array}$ & 2010.3-2011.3 & $\begin{array}{l}\text { Chemotherapy-induced phlebitis } \\
\text { Diseases: esophageal carcinoma29, } \\
\text { gastric cancer47, colon cancer20, } \\
\text { pancreatic cancer7, breast cancer1, } \\
\text { endometrial carcinoma3, gallbladder } 2\end{array}$ & $\begin{array}{l}\text { infrared radiation for } 0.5 \text { hour } \\
\text { once a day }\end{array}$ & $\begin{array}{l}\text { external application of } \\
\text { Ruyijinghuang Powder } \\
\text { combined with winegar } \\
\text { once a day }\end{array}$ & IP & 3 \\
\hline $\begin{array}{l}\text { Zhou } 2013 \\
{[16]}\end{array}$ & $48(24 / 24)$ & $\begin{array}{l}\text { Sex:35/13 } \\
\text { Age:38-75 } M=64\end{array}$ & $\begin{array}{l}2011.3- \\
2012.12\end{array}$ & $\begin{array}{l}\text { Chemotherapy-induced phlebitis } \\
\text { Disease: Gastric cancer17, colorectal } \\
\text { cancer 17, breast cancer13, colon } \\
\text { cancer Phlebitis :E: grade I 20, grade II } \\
16 \text {, grade III } 12\end{array}$ & $\begin{array}{l}\text { external application of } \\
50 \% \mathrm{magnem} \text { sulphate } \\
\left(\mathrm{MgSO}_{4}\right) \text { for } 30 \text { minutes, } 7 \\
\text { days as a course }\end{array}$ & $\begin{array}{l}\text { Ruyijinhuang } \\
\text { powdermixed with } \\
\text { vinegar } \\
\text { once a day, } 7 \text { days as } \\
\text { a course }\end{array}$ & TER & 3 \\
\hline
\end{tabular}

Abbreviations: RCTs, Randomized Controlled Trials; E, Experimental group; C, Control group;NR, no report; IRP, Incidence Rate of phlebitis; TER, Total Effective Rate; AHT,

The average healing time; PRT, pain relief time; IRP, ITP, Incidence time of phlebitis 
Table 1: Basic characteristics of the included studies (continued)

\begin{tabular}{|c|c|c|c|c|c|c|c|c|}
\hline Study & $\begin{array}{l}\text { Sample size } \\
\text { E/C }\end{array}$ & $\begin{array}{l}\text { Sex or Age } \\
\text { E/C } \\
\text { (Mean } \pm \text { SD) } \\
\text { years }\end{array}$ & $\begin{array}{l}\text { Duration } \\
\mathrm{E} / \mathrm{C} \text { (months) }\end{array}$ & $\begin{array}{l}\text { Phlebitis E/C } \\
\text { basic Diseases E/C } \\
\text { Drugs E/C } \\
\text { Phlebitis classification E/C }\end{array}$ & $\begin{array}{l}\text { Topical treatment of } \\
\text { control group }\end{array}$ & $\begin{array}{l}\text { External application of } \\
\text { experimental group }\end{array}$ & $\begin{array}{l}\text { Main } \\
\text { outcomes }\end{array}$ & JADAD \\
\hline $\begin{array}{l}\text { Sun and } \mathrm{Li} \\
2015[17]\end{array}$ & $50(25 / 25)$ & $\begin{array}{l}\text { Sex: E: } 17 / 8 \text { C: } 11 / 14 \\
\text { Age:E:38-75 } \\
(57.3 \pm 7.4) \text { C:36- } \\
77(57.8 \pm 7.6)\end{array}$ & $\begin{array}{l}2010.2- \\
2014.10\end{array}$ & $\begin{array}{l}\text { Chemotherapy-induced phlebitis } \\
\text { E:grade } 13, \text { grade II 5, grade III } 17 \\
\text { C:grade I 2, grade II 4, grade III } \\
19\end{array}$ & $\begin{array}{l}\text { cold compress of } \\
50 \% \mathrm{magnem} \text { sulphate } \\
\left(\mathrm{MgSO}_{4}\right) \text { for } 24 \text { hours, } \\
\text { along with hot compress } \\
\text { for the next } 24 \text { hours }\end{array}$ & $\begin{array}{l}\text { external application of } \\
\text { Ruyijinhuang powder } 9 g \\
\text { mixed with distilled } \\
\text { vinegar } 10 \mathrm{ml} \text { and honey } \\
2 \mathrm{ml} \text { at } 6 \text { hourly intervals }\end{array}$ & TER & 4 \\
\hline $\begin{array}{l}\text { Yu et al } 2010 \\
\text { [18] }\end{array}$ & $88(44 / 44)$ & $\begin{array}{l}\text { Sex: } 41 / 47 \\
\text { Age: } 22-74 M=54.2\end{array}$ & $\begin{array}{l}2007.4- \\
2008.4\end{array}$ & $\begin{array}{l}\text { Chemotherapeutic phlebitis-induced } \\
\text { by indwelling needle Diseases: } \\
\text { gastric cancer18, breast cancer12, } \\
\text { endometrial cancer19, lung } \\
\text { cancer9, liver cancer5, colon } \\
\text { cancer12, pancreatic carcinoma8, } \\
\text { lymphoma5Chemotherapeutic } \\
\text { drugs:Vinorelbine, pharmorubicin }\end{array}$ & $\begin{array}{l}\text { External application of } \\
\text { Hot towel (Temperature } \\
\left.\text { of } 40 \sim 50^{\circ} \mathrm{C}\right)\end{array}$ & $\begin{array}{l}\text { Wet compress of ruyi } \\
\text { jinhuang powder } 50 \mathrm{~g} \\
\text { dissolved in ethanol } 50 \\
\mathrm{ml} \text { for } 2 \mathrm{~h} \text {, once a day, } 7 \\
\text { days as a course }\end{array}$ & TER & 3 \\
\hline $\begin{array}{lrr}\text { You et } & \text { al } \\
2010 & {[19]} & \end{array}$ & $60(30 / 30)$ & $\begin{array}{l}5 / 25 \\
5 / 25 \\
25-78\end{array}$ & $2007.9-2009.6$ & $\begin{array}{l}\text { chemotherapy-induced phlebitis } \\
\text { (Cyclophosphamide, cisplatin, } \\
\text { PTX(paclitaxel) Thiotepa, ADM } \\
\text { doxorubicin) }\end{array}$ & $\begin{array}{l}\text { external application of } \\
\text { hirudoid cream 2-3times } \\
\text { a day }\end{array}$ & $\begin{array}{l}\text { ruyiinhuang powder } \\
\text { mixed with honey at } 0.5 \\
\text { mm thickness } 2-3 \mathrm{~cm} \text { for } \\
12 \mathrm{~h} \text {,twice a day }\end{array}$ & TER & 3 \\
\hline Liu 2011 [21] & $40(20 / 20)$ & $\begin{array}{l}\text { Sex: } 16 / 24 \\
\text { Age:33-75 M= } \\
55.2 \pm 8.3\end{array}$ & $2008.2-2009.10$ & $\begin{array}{l}\text { Chemotherapy-induced phlebitis } \\
\text { Leukemia, multiple myeloma, } \\
\text { lymphoma } \\
\text { E:grade } 16, \text { grade II 11, gradeIII3 } \\
\text { C:grade } 16, \text { grade II 12, gradeIII } 2\end{array}$ & $\begin{array}{l}\text { external application of } \\
50 \% \text { magnem sulphate } \\
\left(\mathrm{MgSO}_{4}\right) \text { for } 12 \mathrm{~h}: \\
\text { Cooperate with external } \\
\text { application of wet Towel } \\
\left(50^{\circ} \mathrm{C}\right) 30 \text { minutes a } \\
\text { time, } 2 \text { times/d }\end{array}$ & $\begin{array}{l}\text { external application of } \\
\text { Ruyijinhuang powder } 5 \mathrm{~g} \\
\text { mixed with honey at } 1 \\
\text { mm thickness for } 10 \\
\text { minutes, twice a day }\end{array}$ & TER & 3 \\
\hline $\begin{array}{l}\text { Meng } 2011 \\
{[22]}\end{array}$ & $60(30 / 30)$ & $\begin{array}{l}\text { Sex: } 7 / 53 \\
\text { Age: } 31-78 \\
M=50.85\end{array}$ & 2010.1-2011.7 & $\begin{array}{l}\text { Chemotherapy-induced phlebitis } \\
\text { chemotherapy drugs: Paclitaxel } \\
\text { cyclophosphamide, Fluorouracil, } \\
\text { Epirubicin. Phlebitis: grade I } 37 \text {, } \\
\text { grade II 19, grade III } 4\end{array}$ & $\begin{array}{l}\text { external application of } \\
50 \% m^{2} \text { anem sulphate } \\
\left(\mathrm{MgSO}_{4}\right), 2-3 \text { times a } \\
\text { day }\end{array}$ & $\begin{array}{l}\text { Ruyijinhuang } \\
\text { powdermixed with dense } \\
\text { tea at } 3 \mathrm{~mm} \text { thickness. }\end{array}$ & TER & 3 \\
\hline
\end{tabular}


Table 1: Basic characteristics of the included studies (continued)

\begin{tabular}{|c|c|c|c|c|c|c|c|c|}
\hline Study & $\begin{array}{l}\text { Sample } \\
\text { size } \\
\text { E/C }\end{array}$ & $\begin{array}{l}\text { Sex or Age } \\
\text { E/C } \\
\text { (Mean } \pm \text { SD) } \\
\text { years }\end{array}$ & $\begin{array}{l}\text { Duration } \\
\mathrm{E} / \mathrm{C} \text { (months) }\end{array}$ & $\begin{array}{l}\text { Phlebitis E/C } \\
\text { basic Diseases E/C } \\
\text { Drugs E/C } \\
\text { Phlebitis classification E/C }\end{array}$ & $\begin{array}{l}\text { Topical treatment of } \\
\text { control group }\end{array}$ & $\begin{array}{l}\text { External application of } \\
\text { experimental group }\end{array}$ & $\begin{array}{l}\text { Main } \\
\text { outcomes }\end{array}$ & JADAD \\
\hline $\begin{array}{l}\text { Liang } 2012 \\
\text { [23] }\end{array}$ & $68(34 / 34)$ & $\begin{array}{l}\text { Sex:38/30 } \\
\text { Age:28-73 } \\
M=51.2\end{array}$ & $\begin{array}{l}2009.1- \\
2011.6\end{array}$ & $\begin{array}{l}\text { Chemotherapeutic phlebitis disease: } \\
\text { nasopharyngeal carcinoma (NPC)15, lung } \\
\text { cancer24, esophageal cancer 14, malignant } \\
\text { lymphoma5, rectal cancer3, breast } \\
\text { cancer7,Phlebitis:E:grade I 15, grade II } \\
\text { 12, gradeIII C:grade I 18, grade II 10, } \\
\text { grade III } 6\end{array}$ & $\begin{array}{l}\text { hot-wet compression } \\
\text { of } 50 \% \text { magnesium } \\
\text { sulphuricum at } 4 \\
\text { hours intervals }, 3 \\
\text { days as a course }\end{array}$ & $\begin{array}{l}\text { external application of } \\
\text { Ruyidinhuang Powder } \\
\text { mixed with honey at } \\
\text { 3mm thickness at } 12 \\
\text { hours intervals, } 3 \text { days } \\
\text { as a course }\end{array}$ & TER & 3 \\
\hline $\begin{array}{l}\text { Kang et al } \\
2013 \text { [24] }\end{array}$ & $59(30 / 29)$ & $\begin{array}{l}\text { Sex: } 41 / 18 \\
\text { Age:32-69 } \\
M=52\end{array}$ & $\begin{array}{l}2012.1- \\
2012.10\end{array}$ & $\begin{array}{l}\text { Chemotherapy non exosmosis phlebitis } \\
\text { Desease:Small cell lung cancer20, } \\
\text { adenocarcinoma15, squamous carcinoma14, } \\
\text { esophageal7, breast cancer3;Chemotherapeutic } \\
\text { drugs: Carboplatin,cisplatin, docetaxel, } \\
\text { paclitaxel, fluorouracil, etoposide, gemcitabine, } \\
\text { doxorubicin }\end{array}$ & $\begin{array}{l}\text { external application } \\
\text { of } 50 \% \text { magnesium } \\
\text { sulphate }\left(\mathrm{MgSO}_{4}\right) 2 \\
\text { times a day }\end{array}$ & $\begin{array}{l}\text { external application of } \\
\text { Ruyijinhuang powder } \\
\text { mixed with sesame oil } \\
50 \% \text { magnesium sulfate } \\
\text { once a day。 }\end{array}$ & TER & 3 \\
\hline $\begin{array}{l}\text { Wang et al } \\
2014 \text { [25] }\end{array}$ & $(60 / 60)$ & $\begin{array}{l}\text { Sex: } 62 / 58 \\
\text { Age: } 36-72\end{array}$ & $\begin{array}{l}2013.10- \\
2014.4\end{array}$ & $\begin{array}{l}\text { Chemotherapy-induced phlebitis Diseases:lung } \\
\text { cancer } 36, \text { gastric cancer } 27 \text {, liver cancer } 20, \\
\text { breast cancer20, colon cancer17 }\end{array}$ & $\begin{array}{l}\text { External application } \\
\text { of } 50 \% \text { magnem } \\
\text { sulphate }\left(\mathrm{MgSO}_{4}\right)\end{array}$ & $\begin{array}{l}\text { external application of } \\
\text { Ruyijinhuang powder } \\
\text { mixed with honey at } \\
3 \mathrm{~mm} \text { thickness for } 72 \\
\text { hours }\end{array}$ & IP & 4 \\
\hline He 2012 [26] & & $\begin{array}{l}\text { Sex:E:28/12 } \\
C: 25 / 15 \\
\text { Age:E:31- } \\
70, M=50.5 \\
C: 22- \\
75, M=48.6\end{array}$ & $\begin{array}{l}2010.8- \\
2011.8\end{array}$ & $\begin{array}{l}\text { Chemotherapy-induced phlebitis Diseases:E:16 } \\
\text { lung cancer, gastric cancer } 8,6 \text { breast cancer, } \\
\text { esophageal cancer3, colon cancer } 7 \text { C:lung } \\
\text { cancer } 15,10 \text { gastric cancer, colorectal cancer } \\
8 \text {, esophageal cancer } 2 \text {, breast cance5 } \\
\text { Chemotherapeutic drugs:Gemcitabine, cisplatin, } \\
\text { 5-fluorouracil, oxaliplatin, doxorubicin }\end{array}$ & $\begin{array}{l}\text { Conventional care } \\
\text { not take preventive } \\
\text { measures }\end{array}$ & $\begin{array}{l}\text { external application of } \\
\text { Ruyijinghuang Powder } \\
\text { mixed with distilled } \\
\text { water, at } 2 \mathrm{~mm} \\
\text { thickness }\end{array}$ & IP & 3 \\
\hline $\begin{array}{l}\text { Wei } \\
{[27]}\end{array}$ & $86(43 / 43)$ & Age:17- 82 & $\begin{array}{l}2006.9- \\
2008.7\end{array}$ & $\begin{array}{l}\text { Chemotherapy-induced phlebitis } \\
\text { Vinorelbine, pirarubicin, mitoxantrone, Docetaxe }\end{array}$ & $\begin{array}{l}\text { external application } \\
\text { of } 50 \% \text { magnem } \\
\text { sulphate }\left(\mathrm{MgSO}_{4}\right) \text { for } \\
4 \text { hours }\end{array}$ & $\begin{array}{l}\text { external application of } \\
\text { Ruyijinhuang powder } \\
\text { mixed with honey at } \\
0.5-1 \mathrm{~mm} \text { thickness for } 4 \\
\text { hours, } 2 \sim 3 d\end{array}$ & IP & 3 \\
\hline
\end{tabular}

Abbreviations: RCTs, Randomized Controlled Trials; E, Experimental group; C, Control group;NR, no report; IRP, Incidence Rate of phlebitis; TER, Total Effective Rate; AHT, The average healing time; PRT, pain relief time; IRP, ITP, Incidence time of phlebitis 
Table 1: Basic characteristics of the included studies (continued)

\begin{tabular}{|c|c|c|c|c|c|c|c|c|}
\hline Study & $\begin{array}{l}\text { Sample size } \\
\text { E/C }\end{array}$ & $\begin{array}{l}\text { Sex or Age } \\
\text { E/C } \\
\text { (Mean } \pm \text { SD) } \\
\text { years }\end{array}$ & $\begin{array}{l}\text { Duration } \\
\mathrm{E} / \mathrm{C} \text { (months) }\end{array}$ & $\begin{array}{l}\text { Phlebitis E/C } \\
\text { basic Diseases E/C } \\
\text { Drugs E/C } \\
\text { Phlebitis classification E/C }\end{array}$ & $\begin{array}{l}\text { Topical treatment of control } \\
\text { group }\end{array}$ & $\begin{array}{l}\text { External application of } \\
\text { experimental group }\end{array}$ & $\begin{array}{l}\text { Main } \\
\text { outcomes }\end{array}$ & JADAD \\
\hline $\begin{array}{lr}\text { Xiong } & \text { and } \\
\text { Yang } & 2013 \\
\text { [29] }\end{array}$ & $74(38 / 36)$ & $\begin{array}{l}\text { Sex: } 36 / 38 \\
\text { Age: } 18-65 \\
M=42.0\end{array}$ & 2011.1-2.13.1 & $\begin{array}{l}\text { PICC-induced Mechanic Phlebitis } \\
\text { breast cancer25, liver cancer } 15 \text {, } \\
\text { cervical cancer13, lung cancer } 1\end{array}$ & $\begin{array}{l}\text { external application of } \\
50 \% \text { magnem sulphate } \\
\left(\mathrm{MgSO}_{4}\right)\end{array}$ & $\begin{array}{l}\text { external application of } \\
\text { Ruyijinhuang powder } \\
\text { mixed with honey at } \\
1 \mathrm{~mm} \text { thickness at } 24 \\
\text { hourly intervals }\end{array}$ & $\begin{array}{l}\text { TER } \\
\text { AHT }\end{array}$ & 4 \\
\hline $\begin{array}{l}\text { Gao and Li } \\
2012[30]\end{array}$ & $108(54 / 54)$ & $\begin{array}{l}\text { Sex:67/41 } \\
\text { Age:4-81 } \\
M=40.6 \pm 7.1\end{array}$ & $2010.2-2011.1$ & $\begin{array}{l}\text { PICC-induced Mechanic } \\
\text { Phlebitis(Cisplatin (DDP) and } \\
\text { fluorouracil) } \\
\text { brain cancer22,nasopharyngeal } \\
\text { carcinoma68, laryngeal cancer } 13 \text {, } \\
\text { carcinoma of the parotid gland5 }\end{array}$ & $\begin{array}{l}\text { Infrared radiation twice a day } \\
\text { for } 3 \text { days }\end{array}$ & $\begin{array}{l}\text { external application of } \\
\text { Ruyijinghuang Powder at } \\
0.5-1.0 \mathrm{~cm} \\
\text { thickniss,twice a day for } \\
3 \text { days }\end{array}$ & IP & 4 \\
\hline $\begin{array}{l}\text { Liu } Y \text { and } \\
\text { Yang LY } \\
2009[31]\end{array}$ & $74(40 / 34)$ & $\begin{array}{l}\text { Sex:E:28/12 } \\
\text { C: } 22 / 12 \\
\text { Age:M=35.2 } \\
C: 33.8\end{array}$ & $2007.1-2009.3$ & PICC-induced Mechanic Phlebitis & $\begin{array}{l}\text { external application of wet } \\
\text { Towel }\left(45-50^{\circ} \mathrm{C}\right) \text { for } 30 \mathrm{~min} \\
\text {,twice a day, } 7 \text { days as a } \\
\text { course }\end{array}$ & $\begin{array}{l}\text { external application of } \\
\text { Ruyijinghuang Powder } \\
\text { combined with sesame oil } \\
\text { once a day, } 7 \text { days as a } \\
\text { course }\end{array}$ & IP & 3 \\
\hline $\begin{array}{l}\text { Duan PP et } \\
\text { al.,2008 [32] }\end{array}$ & $240(120 / 120)$ & $\begin{array}{l}\text { Sex:E:78/42 } \\
\text { C:78/42 } \\
E: 21-89(58 . \\
45 \pm 13.38) \\
C: 16-96(59 . \\
58 \pm 14.85)\end{array}$ & 2005.10-2007.6 & PICC-induced Mechanic Phlebitis & $\begin{array}{l}\text { Conventional care not take } \\
\text { preventive measures }\end{array}$ & $\begin{array}{l}\text { external application of } \\
\text { Ruyijinghuang Powder } \\
\text { once a day for } 3 \text { days }\end{array}$ & $\begin{array}{l}\text { IP } \\
\text { ITP }\end{array}$ & 4 \\
\hline $\begin{array}{l}\text { Zhu et al } \\
2008 \text { [33] }\end{array}$ & $42(21 / 21)$ & $\begin{array}{l}\text { Sex: } 38 / 4 \\
\text { Age:E:2.6 } \pm 3.8 \\
5 \\
C: 82.5 \pm 3.7\end{array}$ & 2005.8-2007.7 & $\begin{array}{l}\text { Phebitis afterPICC catheteterization } \\
\text { in eldedypatients Non-Hodgkin } \\
\text { lymphoma28, myelodysplastic } \\
\text { syndrome9, acute myelogenous } \\
\text { leukemia5 Phebitis :E:grade I5, } \\
\text { grade II } 14, \text { grade III } 2 \text { C:grade } 17 \text {, } \\
\text { grade II } 12, \text { gradeIII } 2\end{array}$ & $\begin{array}{l}\text { Ultrashort wave therapy for } 20 \\
\text { minutes, twice a day }\end{array}$ & $\begin{array}{l}\text { Rnyijinhuang powder } 12 \mathrm{~g} \\
\text { mixed with Warm tea at } \\
1 \mathrm{~mm} \text { thickness for40 } \\
\text { minutes ,2-3times a day }\end{array}$ & TER & 34 \\
\hline
\end{tabular}

The average healing time; PRT, pain relief time; IRP, ITP, Incidence time of phlebitis 
Table 1: Basic characteristics of the included studies (contd)

\begin{tabular}{|c|c|c|c|c|c|c|c|c|}
\hline Study & $\begin{array}{l}\text { Sample } \\
\text { size } \\
\text { E/C }\end{array}$ & $\begin{array}{l}\text { Sex or Age } \\
\mathrm{E} / \mathrm{C} \\
\text { (Mean } \pm \mathrm{SD}) \\
\text { years }\end{array}$ & $\begin{array}{l}\text { Duration } \\
\text { E/C } \\
\text { (months) }\end{array}$ & $\begin{array}{l}\text { Phlebitis E/C } \\
\text { basic Diseases E/C } \\
\text { Drugs E/C } \\
\text { Phlebitis classification E/C }\end{array}$ & $\begin{array}{l}\text { Topical treatment } \\
\text { control group }\end{array}$ & $\begin{array}{l}\text { External application of } \\
\text { experimental group }\end{array}$ & $\begin{array}{l}\text { Main } \\
\text { outcomes }\end{array}$ & JADAD \\
\hline $\begin{array}{l}\text { Li et al } 2007 \\
\text { [34] }\end{array}$ & $62(32 / 30)$ & $\begin{array}{l}\text { Sex:26/36 } \\
\text { Age:13-73 } \\
M=35+2 . \quad 5\end{array}$ & $\begin{array}{l}2004.6- \\
2007.2\end{array}$ & $\begin{array}{l}\text { PICC-induced Mechanic Phlebitis } \\
\text { department of hemopathology and oncology }\end{array}$ & $\begin{array}{l}\text { external application of } \\
50 \% \text { magnem sulphate } \\
\left(\mathrm{MgSO}_{4}\right) \text { for } 12 \mathrm{~h} \text {; Cooperate } \\
\text { with infrared therapeutic } \\
\text { apparatus irradiation, } 30 \\
\text { rain each time, } 2 \text { times a } \\
\text { day. }\end{array}$ & $\begin{array}{l}\text { external application of } \\
\text { Ruyijinhuang powder } \\
\text { mixed with honey for } 24 \\
\text { hours }\end{array}$ & TER ATP & 3 \\
\hline $\begin{array}{lr}\text { Zhu et } & \text { al } \\
2009 & \end{array}$ & $70(36 / 340)$ & $\begin{array}{l}\text { Age:20- } \\
70 \mathrm{M}= \\
42.13\end{array}$ & $\begin{array}{l}2005.1- \\
2008.1\end{array}$ & PICC-induced Mechanic Phlebitis & $\begin{array}{l}\text { external application of } \\
\text { hirudoid cream }, 4 \text { times a } \\
\text { day, for } 2-4 \text { days。 }\end{array}$ & $\begin{array}{l}\text { external application of } \\
\text { Rnyijinhuang powder } 5 \mathrm{~g} \\
\text { mixed with The filtered tea } \\
\text { water } 10 \mathrm{ml} 4 \text { times a day, } \\
\text { for } 2-4 \text { days }\end{array}$ & TER & 3 \\
\hline $\begin{array}{lr}\text { Peng } & \text { and } \\
\text { Wang } & 2015 \\
{[36]}\end{array}$ & & $28 / 4, M=73$ & $\begin{array}{l}2008.1- \\
2010.3\end{array}$ & $\begin{array}{l}\text { PICC-induced phlebitis } \\
\text { Disease:colorectal cancer } \\
\text { Chemotherapy drugs:Oxaliplatin, calcium, } \\
\text { folinate fluorouracil }\end{array}$ & $\begin{array}{l}\text { wet compression of } \\
50 \% \mathrm{magnem} \text { sulphate } \\
\left(\mathrm{MgSO}_{4}\right) \\
\text { infrared radiation for } 20 \\
\text { minutes qn }\end{array}$ & $\begin{array}{l}\text { external application of } \\
\text { Ruyijinhuang powder } 12 \mathrm{~g} \\
\text { mixed with warm water } \\
39-40^{\circ} \mathrm{C} \text { for } 30 \text { minutes, } 3 \\
\text { times a day, } 5 \text { days as a } \\
\text { course }\end{array}$ & TER & 5 \\
\hline $\begin{array}{l}\text { Shi et al } 2011 \\
\text { [37] }\end{array}$ & $73(37 / 36)$ & $\begin{array}{l}\text { Age:20-75 } \\
M=42 \pm 2.5\end{array}$ & $\begin{array}{l}2007.10- \\
2010.10\end{array}$ & PICC-induced Intermittent phlebitis & \multirow{2}{*}{$\begin{array}{l}\text { external application of } \\
\text { Hirudoid ointment combined } \\
\text { with infrared therapy for } 30 \\
\text { minutes, twice a day, } \\
\text { external application of } \\
50 \% \text { magnesium sulphate } \\
\left(\mathrm{MgSO}_{4}\right) ; \\
\text { once a day, } 4 \text { days as a } \\
\text { period of treatment. }\end{array}$} & $\begin{array}{l}\text { external application of } \\
\text { Ruyijinhuang powder } \\
\text { mixed with honey at } 24 \\
\text { hours intervels }\end{array}$ & $\begin{array}{l}\text { TER } \\
\text { ATP }\end{array}$ & 3 \\
\hline Yao 2015 [38] & $86(43 / 43)$ & $\begin{array}{l}\text { Sex:All } \\
\text { woman } \\
\text { Age:30-62 } \\
M=41.6 \pm 10 \\
1\end{array}$ & $\begin{array}{l}2013.6- \\
2014.10\end{array}$ & Infusion phlebitis & & $\begin{array}{l}\text { Ruyijinhuang powder } \\
\text { mixed with vinegar once a } \\
\text { day, } 4 \text { days as a period of } \\
\text { treatment. }\end{array}$ & TER & 3 \\
\hline Yu H2016[40] & $80(40 / 40)$ & $\begin{array}{l}\text { Sex:E:3l/9 } \\
\mathrm{C}: 32 / 8 \\
\text { Age:E:8- } \\
80 M=44 \\
\mathrm{C}: 6- \\
78 \mathrm{M}=47)\end{array}$ & $\begin{array}{l}2013.1- \\
2014.1\end{array}$ & $\begin{array}{l}\text { Infusion phlebitis(fat emulsion, Intravenous } \\
\text { indwelling needle20, Mannitol20,other } \\
\text { drugs26) }\end{array}$ & $\begin{array}{l}\text { External application of } \\
50 \% \text { magnesium } \\
\text { sulphate }\left(\mathrm{MgSO}_{4}\right) 3 \text { times a } \\
\text { day, } 5 \text { days as a course }\end{array}$ & $\begin{array}{l}\text { Ruyijinhuang powder } \\
\text { mixed with sesame oil } \\
\text { twice a day, } \\
5 \text { days as a course }\end{array}$ & TER & 3 \\
\hline
\end{tabular}

Abbreviations: RCTs, Randomized Controlled Trials; E, Experimental group; C, Control group;NR, no report; IRP, Incidence Rate of phlebitis; TER, Total Effective Rate; AHT,

The average healing time; PRT, pain relief time; IRP, ITP, Incidence time of phlebitis 
Table 7: Basic characteristics of the included studies (contd)

\begin{tabular}{|c|c|c|c|c|c|c|c|c|}
\hline Study & $\begin{array}{l}\text { Sample size } \\
\text { E/C }\end{array}$ & $\begin{array}{l}\text { Sex or Age E/C } \\
\text { (Mean } \pm \text { SD) } \\
\text { years }\end{array}$ & $\begin{array}{l}\text { Duration } \\
\text { E/C(months) }\end{array}$ & $\begin{array}{l}\text { Phlebitis E/C } \\
\text { basic Diseases E/C } \\
\text { Drugs E/C } \\
\text { Phlebitis classification E/C }\end{array}$ & $\begin{array}{l}\text { Topical treatment of control } \\
\text { group }\end{array}$ & $\begin{array}{l}\text { External application of } \\
\text { experimental group }\end{array}$ & $\begin{array}{l}\text { Main } \\
\text { outcomes }\end{array}$ & JADAD \\
\hline $\begin{array}{l}\text { Huang } \\
\text { and } \\
2004[39]\end{array}$ & $62(31 / 31)$ & $\begin{array}{l}\text { Sex:25/37 } \\
\text { Age: } 27-80\end{array}$ & $1995.1-2002.3$ & $\begin{array}{l}\text { Infusion phlebitis(Potassium } \\
\text { chloride, nutrient solution and } \\
\text { chemotherapy drugs) } \\
\text { Disease:E:subtotal } \\
\text { gastrectomy7, liver cancer2, } \\
\text { colon cancer 3, acute } \\
\text { pancreatitis4, } \\
\text { Choledocholithotomy7 } 8 \\
\text { postoperative lung cancer } 8 / \\
\text { C:subtotal gastrectomy } 5 \\
\text { Choledocholithotomy } 8 \text {, } \\
\text { postoperative lung cancer 6, } \\
\text { breast cancer 4, kidney } \\
\text { carcinoma liver cance2, } \\
\text { acute intestinal obstruction5 } \\
\text { E:grade I6, grade II } 24, \\
\text { gradeIII } 1 \text { C:grade } 18 \text {, grade II } \\
\text { 22, gradeII } 1\end{array}$ & $\begin{array}{l}\text { external application } \quad \text { of } \\
50 \% \text { magnem sulphate } \\
\left(\mathrm{MgSO}_{4}\right)\end{array}$ & $\begin{array}{l}\text { external application of } \\
\text { Ruyijinhuang powder } \\
\text { mixed with honey at } \\
3 \mathrm{~mm} \text { thickness for } \\
\text { aborve } 12 \text { hours once a } \\
\text { day }\end{array}$ & TER & 4 \\
\hline Wu 2015 [41] & $62(31 / 31)$ & $\begin{array}{l}\text { Sex: } 48 / 14 \\
\text { Age: } 42-89\end{array}$ & 2014.1-2015.7 & $\begin{array}{l}\text { Infusion phlebitis } \\
\text { E:grade } \quad \mid 16, \text { grade II 14, } \\
\text { gradeIII } 1 \\
\text { C:grade } \quad 18, \text { grade II } 22, \\
\text { gradeIII } 1\end{array}$ & $\begin{array}{l}\text { hot-wet compression of } \\
50 \% \text { magnem sulphate } \\
\left(\mathrm{MgSO}_{4}\right)\end{array}$ & $\begin{array}{l}\text { external application of } \\
\text { Ruyijinhuang powder } \\
12 \mathrm{~g} \text { mixed with honey at } \\
1 \mathrm{~mm} \text { thickness for } 3-5 \\
\text { days }\end{array}$ & TER & 3 \\
\hline
\end{tabular}

Abbreviations: RCTs, Randomized Controlled Trials; E, Experimental group; C, Control group;NR, no report; IRP, Incidence Rate of phlebitis; TER, Total Effective Rate; AHT,

The average healing time; PRT, pain relief time; IRP, ITP, Incidence time of phlebitis 
Table 1: Basic characteristics of the included studies (continued)

\begin{tabular}{|c|c|c|c|c|c|c|c|c|}
\hline Study & $\begin{array}{l}\text { Sample size } \\
\text { E/C }\end{array}$ & $\begin{array}{l}\text { Sex or Age E/C } \\
\text { (Mean } \pm \text { SD) } \\
\text { years }\end{array}$ & $\begin{array}{l}\text { Duration } \\
E / C \text { (months) }\end{array}$ & $\begin{array}{l}\text { Phlebitis E/C } \\
\text { basic Diseases E/C } \\
\text { Drugs E/C } \\
\text { Phlebitis classification E/C }\end{array}$ & $\begin{array}{l}\text { Topical treatment of control } \\
\text { group }\end{array}$ & $\begin{array}{l}\text { External application of } \\
\text { experimental group }\end{array}$ & $\begin{array}{l}\text { Main } \\
\text { outcomes }\end{array}$ & $\begin{array}{l}\text { JADA } \\
\text { D }\end{array}$ \\
\hline $\begin{array}{lr}\text { Yan and } \\
\text { Xu } 2015 \\
{[42]}\end{array}$ & $52(26 / 26)$ & $\begin{array}{l}\text { Sex: } 24 / 28 \\
\text { Age:26-74 } \\
M=58.77 \pm 13.18\end{array}$ & $\begin{array}{l}2012.9- \\
2013.6\end{array}$ & $\begin{array}{l}\text { Infusion phlebitis } \\
\text { transfusion drugs:Potassium chloride, } \\
\text { dopamine, fat emulsion ,levofloxacin } \\
\text { Phlebitis:E:grade I 4, grade II } 20 \text {, } \\
\text { gradeIII2 } \\
\text { C:grade I } 6 \text {, grade II 18, grade III } 2\end{array}$ & $\begin{array}{l}\text { external application of } \\
50 \% \text { magnem sulphate }\left(\mathrm{MgSO}_{4}\right) \\
\text { for } \\
120 \text { minutes,twice a day }\end{array}$ & $\begin{array}{l}\text { external application of } \\
\text { Ruyijinhuang powder } \\
\text { mixed with35\%-40\% } \\
\text { ethanol at } 1 \mathrm{~mm} \\
\text { thickness, for } \\
\text { 120minutes, twice a day }\end{array}$ & $\begin{array}{l}\text { TER } \\
\text { ATP }\end{array}$ & 3 \\
\hline $\begin{array}{l}\text { Liu et al } \\
2014 \text { [43] }\end{array}$ & $56(28 / 28)$ & Sex:30/26 & $\begin{array}{l}2011.10- \\
2013.10\end{array}$ & $\begin{array}{l}\text { Infusion phlebitis } \\
\text { Phlebitis:grade } \quad \text { I } 36, \text { grade II 18, } \\
\text { gradeIII }\end{array}$ & $\begin{array}{l}\text { external application of } \\
50 \% \text { magnesium sulphate } \\
\left(\mathrm{MgSO}_{4}\right) 2-3 \text { times a day, } 5 \text { days } \\
\text { as a course }\end{array}$ & $\begin{array}{l}\text { external application of } \\
\text { RuyiJinhuang Powder } \\
15 \mathrm{~g} \text { mixed with Green } \\
\text { tea water at } 2 \mathrm{~mm} \\
\text { thickness for } 24 \text { hours, at } \\
8 \text { hours intervals, } 5 \\
\text { days as a course }\end{array}$ & TER & 3 \\
\hline $\begin{array}{l}\text { Zhang et } \\
\text { al } 2007 \\
{[44]}\end{array}$ & $60(30 / 30)$ & $\begin{array}{l}\text { Sex:36/24 } \\
\text { Age: } 41-78\end{array}$ & $\begin{array}{l}2003.8- \\
2006.8\end{array}$ & $\begin{array}{l}\text { Infusion phlebitis (Potassium chloride } \\
\text { injection, amiodarone, nitric acid } \\
\text { glycerin, dopamine and levofloxacin) }\end{array}$ & $\begin{array}{l}\text { external application of } \\
50 \% \text { magnem sulphate }\left(\mathrm{MgSO}_{4}\right) \\
\text { Cooperate with external } \\
\text { application of wet Towel }(50 \\
\left.{ }^{\circ} \mathrm{C}\right) \text { for } 30 \mathrm{~min} \text {,twice a day } \\
\end{array}$ & $\begin{array}{l}\text { Rnyijinhuang powder } 5 \mathrm{~g} \\
\text { mixed with dense tea, at } \\
3 \mathrm{~mm} \text { thickness for } \\
\text { 10min,twice a day }\end{array}$ & TER & 3 \\
\hline $\begin{array}{l}\text { Liu et } 2012 \\
{[45]}\end{array}$ & $100(50 / 50)$ & $\begin{array}{l}\text { Sex:78/22 } \\
\text { Age:35-71 } \\
M=51\end{array}$ & $\begin{array}{l}2010.1- \\
2012.1\end{array}$ & $\begin{array}{l}\text { Amiodarone induced phlebitis } \\
\text { E:grade I34, grade II 15, } \text { grade III } 3 \\
\text { C:grade I33, grade II 10, } \text { gradeIII } 5\end{array}$ & $\begin{array}{l}\text { external application of } \\
50 \% \text { sulphate } \\
\left(\mathrm{MgSO}_{4}\right)\end{array}$ & $\begin{array}{l}\text { external application of } \\
\text { Ruyijinhuang powder } \\
5 \mathrm{~g} \text { mixed with honey }\end{array}$ & TER & 3 \\
\hline $\begin{array}{lr}\text { Zhang } & \text { and } \\
\text { Zhao } & 2015 \\
{[46]} & \end{array}$ & $60(30 / 30)$ & $\begin{array}{l}\text { Sex:E:16/14 } \\
\text { C: } 14 / 16 \\
\text { Age:E: } 62 \pm 5.4 \\
C: 61 \pm 4.8\end{array}$ & $\begin{array}{l}2014.7- \\
2017.12\end{array}$ & $\begin{array}{l}\text { Amiodarone-induced phelebitis } \\
\text { Phlebitis:E:grade I } 8, \text { grade II 16, } \\
\text { grade II 6 } \\
\text { C:grade I 7, grade II 17, grade II } 6\end{array}$ & $\begin{array}{l}\text { external application of } \\
50 \% \text { magnem sulphate }\left(\mathrm{MgSO}_{4}\right) \\
\text { at } 6 \text { hours intervel }\end{array}$ & $\begin{array}{l}\text { external application of } \\
\text { Ruyijinhuang powder } \\
\text { mixed with honey at } \\
3 \mathrm{~mm} \text { thickness, at } 6 \\
\text { hours intervel }\end{array}$ & $\begin{array}{l}\text { TER } \\
\text { ATP } \\
\text { PRT }\end{array}$ & 4 \\
\hline Dai 2013 [47] & $44(23 / 21)$ & $\begin{array}{l}\text { Sex: } 29 / 15 \\
\text { Age: } 40-83 \\
M=66.25 \pm 12.0 \\
2\end{array}$ & $\begin{array}{l}2011.1- \\
2012.12\end{array}$ & amiodarone-induced phlebitis & $\begin{array}{l}\text { Conventional care not take } \\
\text { preventive measures }\end{array}$ & $\begin{array}{l}\text { Rnyijinhuang powder } 5 \\
\text { g mixed with dense tea } \\
\text { at } 2 \mathrm{~mm} \text { thickness for } 8 \\
-10 \text { hours,twice a day }\end{array}$ & IP & 3 \\
\hline
\end{tabular}

Abbreviations: RCTs, Randomized Controlled Trials; E, Experimental group; C, Control group;NR, no report; IRP, Incidence Rate of phlebitis; TER, Total Effective Rate; AHT,

The average healing time; PRT, pain relief time; IRP, ITP, Incidence time of phlebitis 
Table 9: Basic characteristics of the included studies (contd)

\begin{tabular}{|c|c|c|c|c|c|c|c|c|}
\hline Study & $\begin{array}{l}\text { Sample size } \\
\text { E/C }\end{array}$ & $\begin{array}{l}\text { Sex or Age } E / C \\
\text { (Mean } \pm \text { SD) } \\
\text { years }\end{array}$ & $\begin{array}{l}\text { Duration } \\
E / C \text { (months) }\end{array}$ & $\begin{array}{l}\text { Phlebitis E/C } \\
\text { basic Diseases E/C } \\
\text { Drugs E/C } \\
\text { Phlebitis classification E/C }\end{array}$ & $\begin{array}{l}\text { Topical treatment of control } \\
\text { group }\end{array}$ & $\begin{array}{l}\text { External application of } \\
\text { experimental group }\end{array}$ & $\begin{array}{l}\text { Main } \\
\text { outcomes }\end{array}$ & JADAD \\
\hline $\begin{array}{l}\text { Huo and } \\
\text { Chang } \\
2013[48]\end{array}$ & $60(30 / 30)$ & $\begin{array}{l}\text { Sex:33/27 } \\
\text { Age:35-78M= } \\
54.23 \pm 5.52\end{array}$ & $\begin{array}{l}2008.10- \\
2012.10\end{array}$ & amiodarone-induced phlebitis & $\begin{array}{l}\text { external application of } \\
50 \% \text { magnesium sulphate } \\
\left(\mathrm{MgSO}_{4}\right) \text {; } \\
30 \text { minutes each time, } 3 \text { times a } \\
\text { day, } 3 \text { days as a period of } \\
\text { treatment. }\end{array}$ & $\begin{array}{l}\text { Ruyi j inhuang Powder } \\
15 \mathrm{~g} \text { mixed } \\
\text { with vinegar at } 0.5 \mathrm{~mm} \\
\text { thickness }\end{array}$ & TER & 3 \\
\hline $\begin{array}{l}\text { Chen et al } \\
2013 \text { [49] }\end{array}$ & $60(30 / 30)$ & $\begin{array}{l}\text { Sex:E:21/9 } \\
\text { C:21/9 } \\
\text { Age:E:36- } \\
78, M=67 \\
\text { C: } 32-85, M=70\end{array}$ & $\begin{array}{l}2011.1- \\
2012.2\end{array}$ & 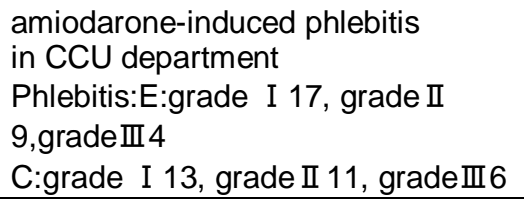 & $\begin{array}{l}\text { external application of } 50 \% \\
\text { magnem sulphate }\left(\mathrm{MgSO}_{4}\right) \text { : } \\
\text { twice a day }\end{array}$ & $\begin{array}{l}\text { external application of } \\
\text { Ruyijinhuang powder } \\
\text { mixed with } 75 \% \text { ethanol at } \\
3 \mathrm{~mm} \text { thickness for } 8-12 \\
\text { hours twice a day }\end{array}$ & $\begin{array}{l}\text { TER } \\
\text { ATP } \\
\text { PRT }\end{array}$ & 3 \\
\hline $\begin{array}{l}\text { Fu } 2016 \\
{[50]}\end{array}$ & $146(82 / 64)$ & $\begin{array}{l}\text { Sex: } E: 34 / 30 \\
\text { C: } 43 / 39\end{array}$ & $\begin{array}{l}2015.1- \\
2015.12\end{array}$ & $\begin{array}{l}\text { sodium } \beta \text {-seven-induced phlebitis } \\
\text { E:grade I } 36, \text { grade II } 27, \text { grade III } \\
19 \\
\text { C:grade I } 28, \text { grade II } 22, \text { grade } \\
\text { III } 14\end{array}$ & $\begin{array}{l}\text { wet compress of } 50 \% \\
\text { magnesium sulphuricum for } 1 \\
\text { hours, 3times a day, } 5 \text { days as } \\
\text { a course }\end{array}$ & $\begin{array}{l}\text { external application of } \\
\text { RuyiJinhuang Powder } \\
\text { tuned into a paste with } \\
\text { honey at } 2 \mathrm{~mm} \text { thickness } \\
\text { for } 22 \text { hours, once a day, } \\
5 \text { days as a course }\end{array}$ & TER & 3 \\
\hline $\begin{array}{l}\mathrm{Li} \text { et al } \\
2013[51]\end{array}$ & $80(40 / 40)$ & $\begin{array}{l}\text { Sex:38/42 } \\
\text { Age:M=52.3 }\end{array}$ & $\begin{array}{l}2011.3- \\
2012.9\end{array}$ & $\begin{array}{l}\text { fat emulsion-induced phlebitis } \\
\text { diseases:severe acute } \\
\text { pancreatitis62, tumor14, } \\
\text { malnutrition4 }\end{array}$ & $\begin{array}{l}\text { external application of } \\
50 \% \text { magnesium sulphate } \\
\left(\mathrm{MgSO}_{4}\right) 50 \mathrm{~g} \text { dissolved in } \\
100 \mathrm{~mL} \text { warm water }\left(40-45^{\circ} \mathrm{C}\right) \\
\text { cooperate with microwave } \\
\text { physiotherapy } 15-30 \text { minutes, } 2-3 \\
\text { times a day } 10-20 \text { min each time }\end{array}$ & $\begin{array}{l}\text { hydropathic compress of } \\
\text { Ruyi Jinhuang powder } \\
7 \text { days for a course of } \\
\text { treatment }\end{array}$ & $\begin{array}{l}\text { TER } \\
\text { ATP } \\
\text { PRT }\end{array}$ & 3 \\
\hline $\begin{array}{l}\text { Yang } \\
2012[52]\end{array}$ & $75(37 / 38)$ & $\begin{array}{l}\text { Sex:52/23 } \\
\text { Age: } 35-78\end{array}$ & $\begin{array}{l}2010.1- \\
2012.5\end{array}$ & $\begin{array}{l}\text { mannitol-induced phlebitis } \\
\text { Phlebitis:E:grade I 27, grade II 10, } \\
\text { gradeIII0 C:grade I } 30 \text {, grade II } 8 \text {, } \\
\text { grade III } 0\end{array}$ & $\begin{array}{l}\text { external application of } \\
25 \% \text { magnem sulphate }\left(\mathrm{MgSO}_{4}\right)\end{array}$ & $\begin{array}{l}\text { external application of } \\
\text { Ruyijinhuang powder } 2- \\
3 \mathrm{~g} \text { mixed with vinegar at } \\
2 \mathrm{~mm} \text { thickness }\end{array}$ & TER & 3 \\
\hline $\begin{array}{l}\text { Yang } \\
2012[52]\end{array}$ & $75(37 / 38)$ & $\begin{array}{l}\text { Sex:52/23 } \\
\text { Age: } 35-78\end{array}$ & $\begin{array}{l}2010.1- \\
2012.5\end{array}$ & $\begin{array}{l}\text { mannitol-induced phlebitis } \\
\text { Phlebitis:E:grade I 27, grade II 10, } \\
\text { grade III0 C:grade I } 30 \text {, grade II } 8 \text {, } \\
\text { grade III } 0\end{array}$ & $\begin{array}{l}\text { external application of } \\
25 \% \text { magnem sulphate }\left(\mathrm{MgSO}_{4}\right)\end{array}$ & $\begin{array}{l}\text { external application of } \\
\text { Ruyijinhuang powder } 2 \text { - } \\
3 \mathrm{~g} \text { mixed with vinegar at } \\
2 \mathrm{~mm} \text { thickness }\end{array}$ & TER & 3 \\
\hline
\end{tabular}

Abbreviations: RCTs, Randomized Controlled Trials; E, Experimental group; C, Control group;NR, no report; IRP, Incidence Rate of phlebitis; TER, Total Effective Rate; AHT,

The average healing time; PRT, pain relief time; IRP, ITP, Incidence time of phlebitis 
Table 1: Basic characteristics of the included studies (continued)

\begin{tabular}{|c|c|c|c|c|c|c|c|c|}
\hline Study & $\begin{array}{l}\text { Sample size } \\
\text { E/C }\end{array}$ & $\begin{array}{l}\text { Sex or Age } E / C \\
\text { (Mean } \pm \text { SD) } \\
\text { years }\end{array}$ & $\begin{array}{l}\text { Duration } \\
E / C \text { (months) }\end{array}$ & $\begin{array}{l}\text { Phlebitis E/C } \\
\text { basic Diseases E/C } \\
\text { Drugs E/C } \\
\text { Phlebitis classification E/C }\end{array}$ & $\begin{array}{l}\text { Topical treatment of control } \\
\text { group }\end{array}$ & $\begin{array}{l}\text { External application of } \\
\text { experimental group }\end{array}$ & $\begin{array}{l}\text { Main } \\
\text { outcomes }\end{array}$ & JADAD \\
\hline $\begin{array}{l}\text { Tang G } \\
\text { and Meng } \\
\text { JH } \\
2012[53]\end{array}$ & $60(30 / 30)$ & $\begin{array}{l}\text { Sex:36/24 } \\
\text { Age:37-72 } \\
M=68 \pm 9\end{array}$ & $\begin{array}{l}2007.3- \\
2010.9\end{array}$ & $\begin{array}{l}\text { Gadopentetate dimeglumine - } \\
\text { induced phlebitis } \\
\text { grade } 140, \text { grade II 18, gradeIII } 2\end{array}$ & $\begin{array}{l}\text { external application of } \\
50 \% \text { magnem sulphate }\left(\mathrm{MgSO}_{4}\right) \\
\text { twice a day }\end{array}$ & $\begin{array}{l}\text { external application of } \\
\text { Ruyijinghuang Powder } \\
\text { combined with } \\
\text { glycerinum, twice a day }\end{array}$ & TER & 3 \\
\hline $\begin{array}{l}\mathrm{Li} \text { et al } \\
2010[55]\end{array}$ & $100(50 / 50)$ & $\begin{array}{l}E: 75-82 M=78.5 \pm \\
3.2) \\
C: 75-84 M= \\
(79.9 \pm 2.9)\end{array}$ & $\begin{array}{l}2008.10- \\
2009.6\end{array}$ & $\begin{array}{l}\text { Alprostadil-induced superficial } \\
\text { phlebitis }\end{array}$ & $\begin{array}{l}\text { external application of Dermlin } \\
\text { dressing, once a day }\end{array}$ & $\begin{array}{l}\text { external application of } \\
\text { Ruyijinghuang Powder } \\
12 \mathrm{~g} \text { combined with } \\
\text { glycerinum } 20 \mathrm{ml} \text { for } 30 \\
\text { minutes, } 2-3 \text { times a day } \\
3 \text { days as a course }\end{array}$ & TER & 3 \\
\hline $\begin{array}{l}\text { Mei } 2010 \\
{[56]}\end{array}$ & $100(50 / 50)$ & $\begin{array}{l}\text { Sex:48/52 } \\
\text { Age:63-84 }\end{array}$ & $\begin{array}{l}2009.3- \\
2009.12\end{array}$ & $\begin{array}{l}\text { superficial phlebitis } \\
\text { E:grade I, grade II 17, grade III } 6 \\
\text { C:gradel } 30, \text { grade I 17例, grade } \\
\text { II } 3\end{array}$ & $\begin{array}{l}\text { external application of Dermlin } \\
\text { dressing, once a day }\end{array}$ & $\begin{array}{l}\text { external application of } \\
\text { Ruyijinghuang Powder } \\
12 \mathrm{~g} \text { combined with } \\
\text { glycerinum } 20 \mathrm{~mL} \text {, at } 0.3 \\
\text { cm thickniss, 2-3times a } \\
\text { day, } 7 \text { days as a course }\end{array}$ & TER & 4 \\
\hline $\begin{array}{l}\text { Zhang } \\
\text { and Wang } \\
2013[57]\end{array}$ & $50(25 / 25)$ & $\begin{array}{l}\text { Sex: } 28 / 22 \\
\text { Age: } 50-90 \mathrm{M}= \\
70\end{array}$ & $\begin{array}{l}2012.8- \\
2012.12\end{array}$ & indwelling needle- induced phlebitis & $\begin{array}{l}\text { Conventional care not take } \\
\text { preventive measures }\end{array}$ & $\begin{array}{l}\text { external application of } \\
\text { Ruyijinhuang powder } \\
\text { mixed with honey for } 12 \\
\text { hours, twice a day }\end{array}$ & IP & 3 \\
\hline $\begin{array}{l}\text { Zhou et al } \\
2012[58]\end{array}$ & $128(64 / 64)$ & $\begin{array}{l}\text { Age:E:28- } \\
70(52.22+12.98) \\
\text { C:26- } \\
72(52.47+13.32)\end{array}$ & $\begin{array}{l}2009.1- \\
2010.10\end{array}$ & $\begin{array}{l}\text { indwelling needle- induced phlebitis } \\
\text { desease:cervical carcinom } \\
\text { Chemotherapeutic drugs: } \\
\text { Fluorouracil }\end{array}$ & $\begin{array}{l}\text { Conventional care not take } \\
\text { preventive measures }\end{array}$ & $\begin{array}{l}\text { external application of } \\
\text { RuyiJinhuang Powder } \\
15 \mathrm{~g} \text { mixed with honey for } \\
24 \text { hours, } 5 \text { days as a } \\
\text { course }\end{array}$ & $\begin{array}{l}\text { IP } \\
\text { ITP }\end{array}$ & 4 \\
\hline $\begin{array}{l}\text { Yu et al } \\
2010[59]\end{array}$ & & $\begin{array}{l}\text { Sex:30/18 } \\
\text { Age: } 25-83\end{array}$ & $\begin{array}{l}2008.1- \\
2009.12\end{array}$ & $\begin{array}{l}\text { indwelling needle-induced phlebitis } \\
\text { phlebitis:grade I } 35, \text { grade II } 13\end{array}$ & $\begin{array}{l}\text { external application of } \\
50 \% \text { magnem sulphate }\left(\mathrm{MgSO}_{4}\right) \\
\text { : Cooperate with external } \\
\text { application of hot-water bag ,at } \\
4 \text { hours intervels, } 5 \text { day as a } \\
\text { course }\end{array}$ & $\begin{array}{l}\text { external application of } \\
\text { Ruyijinhuang powder } \\
\text { mixed with vinegar at } 0.2- \\
0.4 \mathrm{~cm} \text { thickness, once a } \\
\text { day }, 5 \text { day as a course }\end{array}$ & TER & 3 \\
\hline $\begin{array}{l}\text { Xin } 2012 \\
{[60]}\end{array}$ & $60(30 / 30)$ & $\begin{array}{l}\text { Sex:32/28 } \\
\text { Age:26-82 } \\
M=54 \pm 12.5\end{array}$ & $\begin{array}{l}2010.1- \\
2011.1\end{array}$ & $\begin{array}{l}\text { indwelling needle- induced phlebitis } \\
\text { cardiothoracic surgery department }\end{array}$ & $\begin{array}{l}\text { external application of } \\
50 \% \text { magnem sulphate }\left(\mathrm{MgSO}_{4}\right) \\
\text {, Cooperate with external } \\
\text { application of hot-water bag }(50 \\
\left.{ }^{\circ} \mathrm{C}\right), 4 \text { times a day, } 7 \text { days as a } \\
\text { course }\end{array}$ & $\begin{array}{l}\text { Ruyijinhuang powder } 9 \mathrm{~g} \\
\text { mixed with distilled } \\
\text { vinegar } 20 \mathrm{ml} \text { at } 12 \\
\text { hourly intervals, } 7 \text { days } \\
\text { as a course }\end{array}$ & TER & 4 \\
\hline
\end{tabular}


Table 1: Basic characteristics of the included studies (continued)

\begin{tabular}{|c|c|c|c|c|c|c|c|c|}
\hline Study & $\begin{array}{l}\text { Sample size } \\
\text { E/C }\end{array}$ & $\begin{array}{l}\text { Sex or Age } E / C \\
\text { (Mean } \quad \pm \quad S D) \\
\text { years }\end{array}$ & $\begin{array}{l}\text { Duration } \\
E / C \text { (months) }\end{array}$ & $\begin{array}{l}\text { Phlebitis E/C } \\
\text { basic Diseases E/C } \\
\text { Drugs E/C } \\
\text { Phlebitis classification E/C }\end{array}$ & $\begin{array}{l}\text { Topical treatment of control } \\
\text { group }\end{array}$ & $\begin{array}{l}\text { External application of } \\
\text { experimental group }\end{array}$ & $\begin{array}{l}\text { Main } \\
\text { outcomes }\end{array}$ & JADAD \\
\hline $\begin{array}{l}\text { Xu et al } \\
2011[61]\end{array}$ & $120(60 / 60)$ & $\begin{array}{l}40 / 20(2-84 \mathrm{M}= \\
43) \\
38 / 22(4- \\
80 \mathrm{M}=42)\end{array}$ & $\begin{array}{l}2008.6- \\
2010.6\end{array}$ & $\begin{array}{l}\text { indwelling needle- induced phlebitis } \\
\text { E:Swelling type } 37 \text {, vascular } \\
\text { sclerosis type } 21 \text {, necrosis Type } 2 \\
\text { C:Swelling type } 36 \text {, vascular } \\
\text { sclerosis type } 22 \text {, necrosis type2 }\end{array}$ & $\begin{array}{l}0.5 \% \text { povidone iodine solution } \\
\text { cotton swab sterile needle, } \\
\text { anisodamine } 10 \mathrm{mg} \text { plus NS } \\
\text { soaked } 10 \mathrm{ml} \text { sterile gauze to } \\
\text { cover, cover with plastic wrap. } \\
30 \text { minutes each time, } 2 \text { times a } \\
\text { day, } 5 \text { days as a period of } \\
\text { treatment. }\end{array}$ & $\begin{array}{l}\text { Ruyi jinhuang Powder } \\
\text { mixedwith ethyl alcohol } \\
\text { (applied the Ruyi } \\
\text { jinhuang } \\
\text { Powder which had been } \\
\text { put on the gauze evenly } \\
\text { to the damaged skin } \\
\text { according to its Size)as } \\
\text { well as TDP based on } \\
\text { sys temic, twice per day } \\
30 \text { minutes each time, } \\
5 \text { days as a course. }\end{array}$ & TER & 3 \\
\hline $\begin{array}{l}\text { Song et al } \\
2014 \text { [62] }\end{array}$ & $32(16 / 16)$ & $\begin{array}{l}\text { Sex:E:20/17 } \\
\text { C: } 21 / 15 \\
\text { Age:E:60-72.5 } \\
C: 60.5-71.9\end{array}$ & $\begin{array}{l}2012.5- \\
2014.1\end{array}$ & indwelling needle- induced phlebitis & $\begin{array}{l}\text { external application of } \\
50 \% \text { magnesium sulphate } \\
\left(\mathrm{MgSO}_{4}\right) 50 \mathrm{~g} \text { dissolved in } \\
100 \mathrm{~mL} \text { warm water }\left(40-45^{\circ} \mathrm{C}\right) 3 \\
\text { times a day } 10-20 \text { min each } \\
\text { time, } 4-6 \text { days as a course }\end{array}$ & $\begin{array}{l}\text { Ruyijinhuang powder } \\
\text { mixed with sesame oil } \\
\text { twice a day, } \\
4-6 \text { days as a course }\end{array}$ & TER & 3 \\
\hline $\begin{array}{l}\text { Liu and } \\
\text { Zhou } \\
2012 \text { [63] }\end{array}$ & $46(23 / 23)$ & $\begin{array}{l}\text { Sex:30/16 } \\
\text { Age:22-85 }\end{array}$ & $\begin{array}{l}2010.1- \\
2011.8\end{array}$ & $\begin{array}{l}\text { indwelling needle- induced phlebitis } \\
\text { (breast cancer } 6 \text {, lung cancer } 18 \text {, } \\
\text { colorectal cancer } 10 / \\
\text { cervical cancer } 8 \text {, brain tumor } 2 \text {, } \\
\text { non-Hodgkin's lymphoma 2) }\end{array}$ & $\begin{array}{l}\text { external application of } \\
50 \% \text { magnem sulphate }\left(\mathrm{MgSO}_{4}\right) \\
\text { for } 2 \mathrm{~h}, 3 \text { times a day,3days as a } \\
\text { course }\end{array}$ & $\begin{array}{l}\text { Ruyijinhuang } \\
\text { powdermixed with honey } \\
\text { for } 12 \mathrm{~h} \text {, once a day, } \\
\text { 3days as a course }\end{array}$ & TER & 3 \\
\hline
\end{tabular}

The average healing time; PRT, pain relief time; IRP, ITP, Incidence time of phlebitis 
Thus, randomized-effect model should be used for statistical analysis. The combined effects showed that there was some beneficial evidence regarding the effect on reducing incidence of phlebitis after external application of Ruyi jinhuang powder versus conventional therapy $(\mathrm{RR}=0.32,95 \% \mathrm{Cl}=0.24$ and 0.42 , and $p<$ 0.0001) (Figure 3).

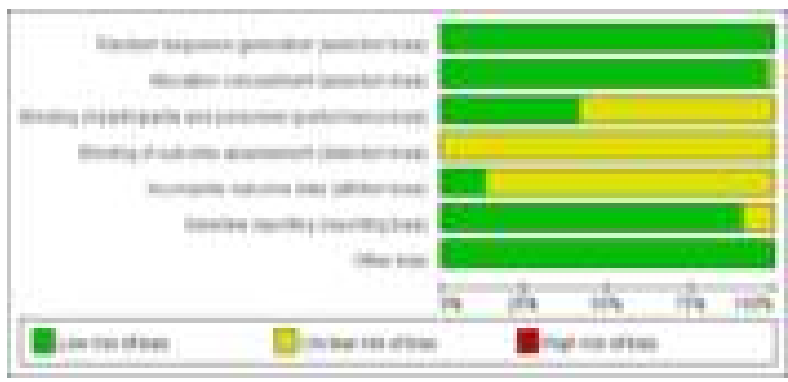

Figure 3: Meta-analysis of the incidence rate of phlebitis Ruyi jinghuang powder versus conventional therapy based on the same intervention strategies in preventing phlebitis. $\mathrm{Cl}$ indicates confidence interval

Total effectiveness rate of Ruyi Jinhuang powder versus conventional therapy in treating phlebitis

39 RCTs contained 2701 patients illustrated the results. The experimental group (1364 trials) and control groups (1337 trials) received Ruyi Jinhuang powder and conventional therapy, respectively. The 39 trials yielded significant heterogeneity $\left(X^{2}=171.17, P<0.0001, P=78 \%\right)$. Thus, random-effect model should be used for statistical analysis.

The aggregated results indicated that comparison revealed significant differences in total effectiveness rate of Ruyi Jinhuang powder versus conventional therapy groups $(\mathrm{RR}=1.27$, $95 \% \mathrm{Cl}=1.19,1.36$, and $P<0.0001)$.

Significant differences were also found between subgroups of chemotherapy-induced phlebitis $(\mathrm{RR}=1.19,95 \% \mathrm{Cl}=1.10,1.27$, and $p<$ 0.0001). PICC-induced phlebitis $(R R=1.65,95$ $\% \mathrm{Cl}=1.39,1.96$, and $p<0.00001)$. infusion phlebitis $(\mathrm{RR}=1.21,95 \% \mathrm{Cl}=1.10,1.33$, and $p$ $=0.0001)$ and indwelling needle-induced phlebitis $(\mathrm{RR}=1.28,95 \% \mathrm{Cl}=1.08,1.52$, and $p$ $=0.007$; Figure 4 to Figure 6 ).

\section{Secondary outcomes}

\section{Average healing time}

Seven RCTs consisting of 461 patients illustrated the results. The experimental group included 233 trials, and the control groups included 228 trials. The 7 trials shown significant heterogeneity $\left(x^{2}\right.$
=303.59, $\left.p<0.00001, P^{2}=98 \%\right)$ compared to conventional therapy. External application of Ruyi jinhuang powder exerted a superior effect on the average healing time using the randomeffect model $(\mathrm{MD}=-32.17,95 \% \mathrm{Cl}=[-48.39$, 15.94], and $P=0.0001$ ) (Figure 7).

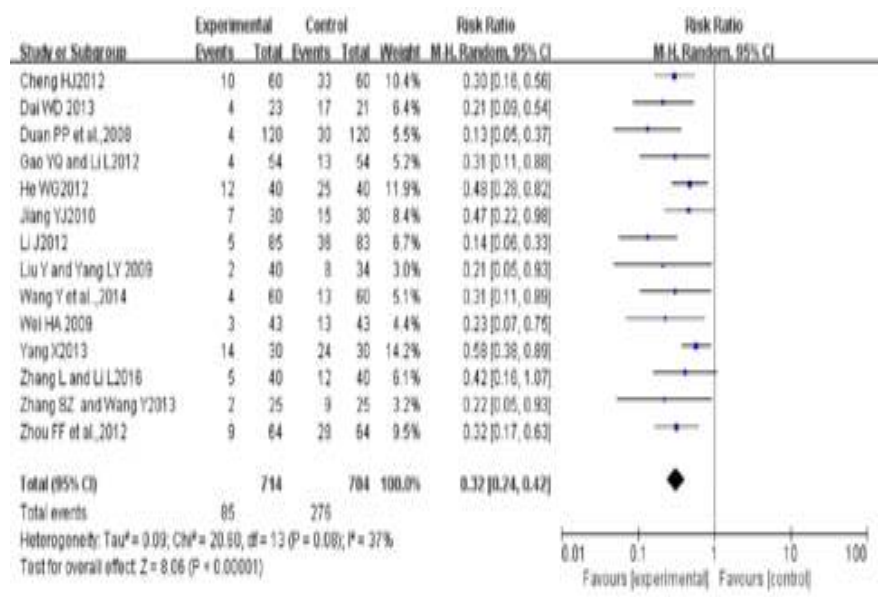

Figure 4: Meta-analysis of the total effectiveness rate of external application of Ruyi jinghuang powder versus conventional therapy based on the same intervention strategies. $\mathrm{Cl}$ indicates confidence interval.

\section{A: Chemotherapy-induced phlebitis}

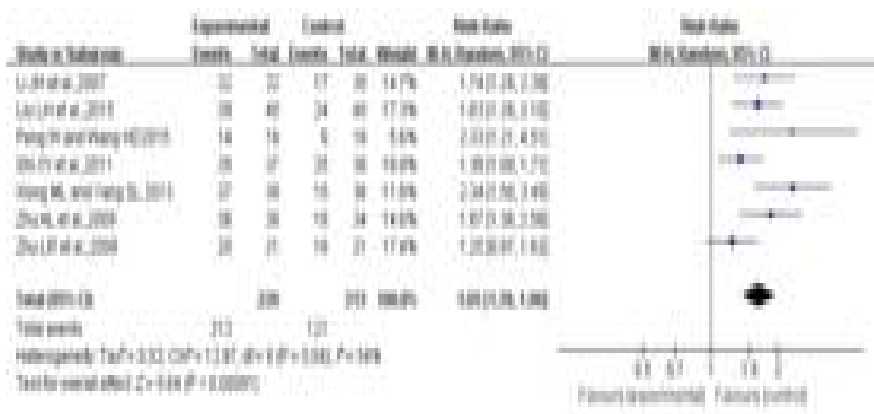

\section{B: PICC-induced phlebitis}

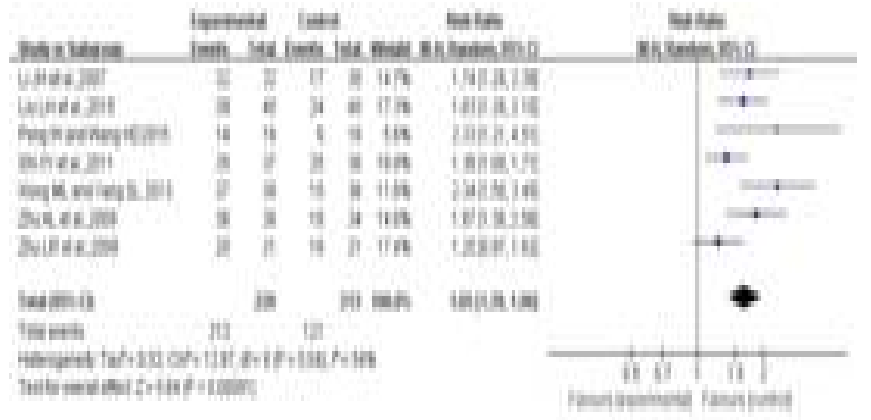

Figure 5: Meta-analysis of the total effectiveness rate of external application of Ruyi jinghuang powder versus conventional therapy based on the same intervention strategies. $\mathrm{Cl}$ indicates confidence interval. 


\section{C: Infusion phlebitis}

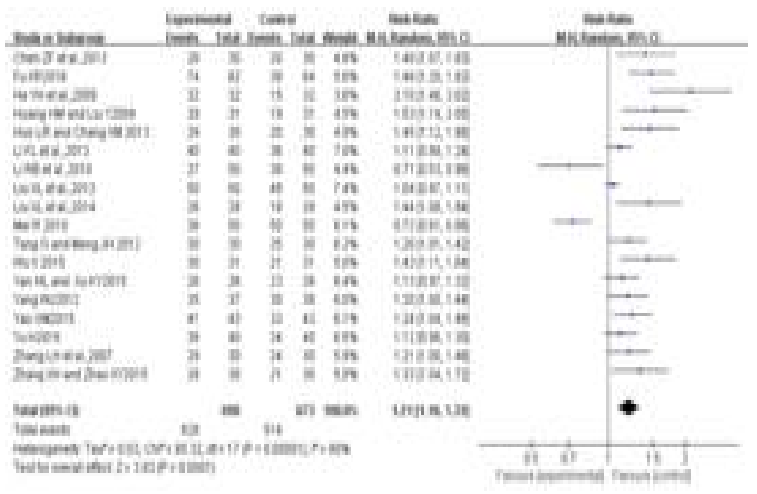

\section{D: Indwelling needle-induced phlebitis}

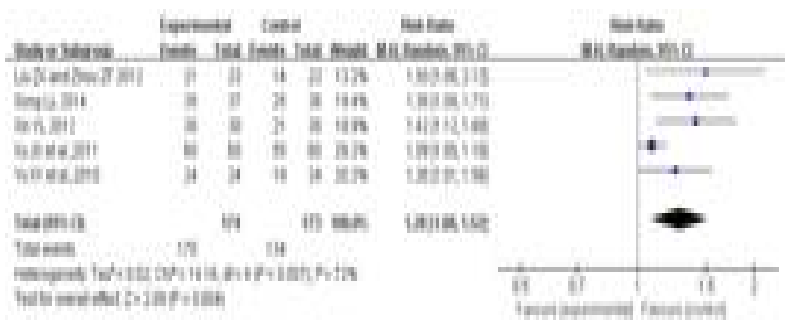

Figure 6: Meta-analysis of the total effectiveness rate of external application of Ruyi jinghuang powder versus conventional therapy based on the same intervention strategies. $\mathrm{Cl}$ indicates confidence interval.

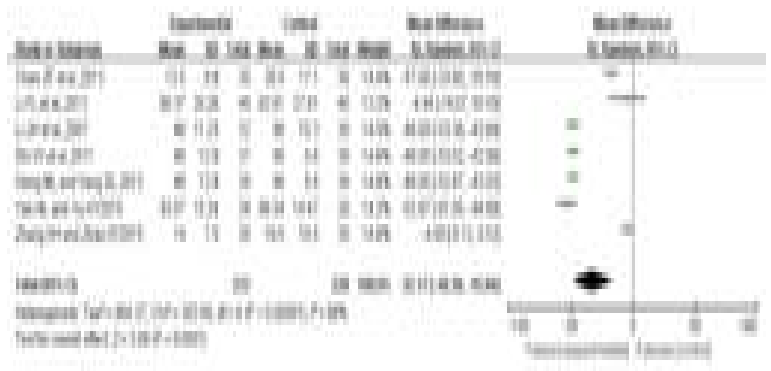

Figure 7: Meta-analysis of the average healing time of external application of Ruyi jinghuang powder versus conventional therapy

\section{Pain relief time}

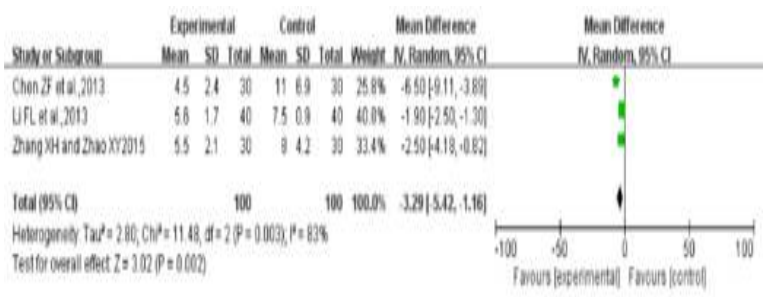

Three studies contained 200 patients reported pain relief time, the experimental group included 100 trials and the control group included 100 trials. The 3 trials yielded significant heterogeneity $\left(X^{2}=11.48, P=0.003, P^{2}=83 \%\right)$, the results of meta-analysis using the randomizedeffect model indicated significant difference on pain relief time of phlebitis $(\mathrm{MD}=-3.29,95 \% \mathrm{Cl}$ $=[-5.42,-1.16], P=0.002)$, as illustrated in Figure 8.

Figure 8: Meta-analysis of the average healing time of external application of Ruyi Jinghuang powder versus conventional therapy

\section{Incidence time of phlebitis}

Two RCTs consisting of 368 patients illustrated the results. The experimental group included 184 trials and control group included 184 trials. These 2 trials yielded significant heterogeneity $\left(x^{2}=\right.$ $17.44, p<0.0001, P^{2}=94 \%$ ), but result of metaanalysis indicated there was no statistical significance in incidence time of phlebitis (MD = $0.62,95 \% \mathrm{Cl}=[-1.76,0.52], p=0.29)$, as demonstrated in Figure 9.

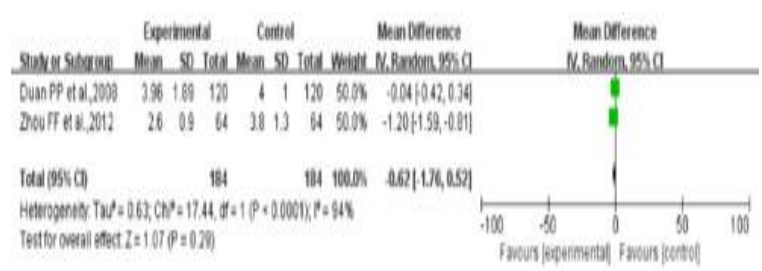

Figure 9: Meta-analysis of the incidence time of phlebitis of external application of Ruyijinghuang Powder versus conventional therapy

\section{Adverse events}

Only one patient reported adverse event as rash reaction with topical application of Ruyi Jinhuang powder mixed with distilled water, manifested as local skin irritation, itching, neck visible red rash, after removing the topical drug immediately and intramuscular injection of diphenhydramine 20 $\mathrm{mg}$ was given at the same time, rash symptoms relieved within 30 min [26]. No other study has reported adverse events induced by Ruyi jinhuang powder. However, the effect of Ruyi jinhuang powder expression still need to further standardize and clear, usage of unknown "for external use" has become the underlying causes for the incidence of adverse reactions $[27,28]$.

\section{Sensitivity analysis}

Using the leave-one-out approach, sensitivity analysis demonstrated the finding was reliable and independent, no significant change was noted in the direction of the combined estimates after the study removal, suggesting that the meta-analysis was robust and the data was accurate. 


\section{Publication bias assessment}

In this review, although all of included literatures were the comparisons of the Chinese literature, the funnel plots of incidence of phlebitis, total effectiveness rate in treating phlebitis, average healing time, pain relief time, incidence time of phlebitis performed included 14 RCTs, 39 RCTs, 7 RCTs, 3 RCTs and 2 RCTs, respectively (Figure 8-10).The use of funnel plots of pain relief time and incidence time of phlebitis was limited because of the sample size. Regarding these studies of Ruyi Jinhuang powder for phlebitis, the publication bias was small because the spots were substantially symmetric, and none of the studies lies outside the limits of the $95 \% \mathrm{Cl}$. However, caution is advised in interpreting the results of publication bias of average hospitalization time after operation because of a small subset of studies. Consequently, the publication bias probably occurs in this metaanalysis.

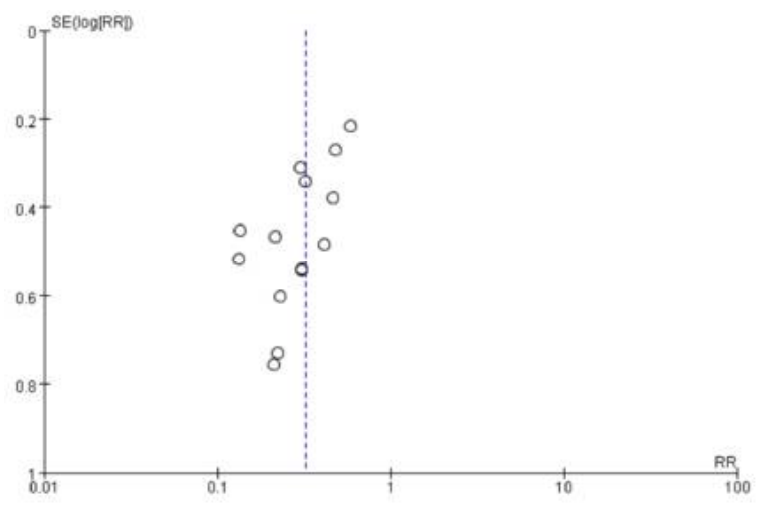

Figure 10: Funnel plot of the incidence rate of phlebitis with Ruyijinghuang Powder versus conventional therapy based on the same intervention strategies in preventing phlebitis

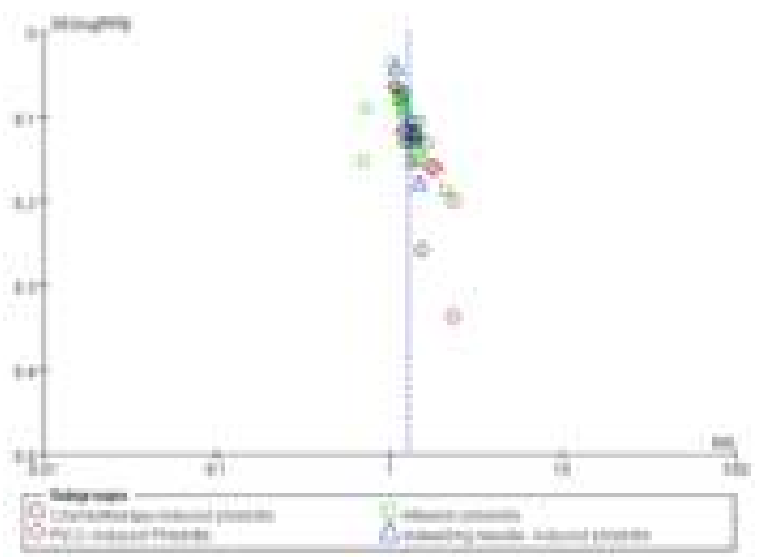

Figure 11: Funnel plot of the total effectiveness rate of phlebitis with external application of Ruyijinghuang Powder versus conventional therapy based on the same intervention strategies

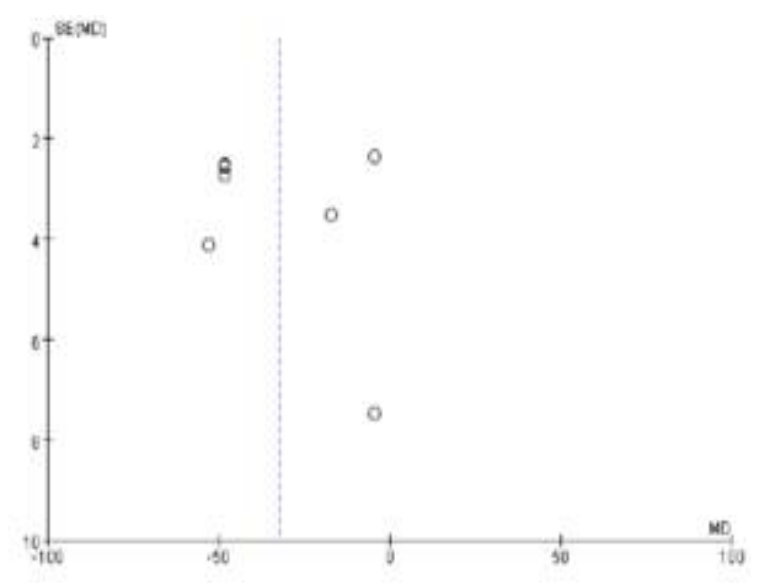

Figure 12: Funnel plot of the average healing time of phlebitis with external application of Ruyi Jinghuang powder versus conventional therapy based on the same intervention strategies

\section{DISCUSSION}

\section{Summary of evidence}

This systematic review searched a wide variety of electronic databases for relevant articles. A total of 53 RCTs were identified, a detailed subgroup analysis based on different comparisons revealed the clinical outcome of phlebitis. Despite the fact that most of the trials had small sample sizes and poor methodological quality, analysis of the pooled data showed a consistently superior effect of Ruyi Jinhuang powder in terms of increasing the total effectiveness rate, which is in accordance with the prior two meta-analyses [8,9]. Whereas, reducing the incidence of phlebitis, which was not consistent with previous study [9], one important probable cause was the expanded sample size. Furthermore, our study showed that Ruyi jinhuang powder could even lead to a shorter postoperative recovery time by decreasing average healing time and pain relief time of phlebitis compared to the control groups, which were not mentioned in the two prior studies. However, result of meta-analysis indicated there was no statistically significant difference in incidence time of phlebitis. Moreover, no patients dropped out of their trials due to adverse effects, suggesting that Ruyi Jinhuang powder was relatively safe for clinical use.

\section{Strengths of this review}

The prior two meta-analyses of Ruyi Jinhuang powder demonstrated significant benefits compared with $50 \%$ magnesium sulfate and no adverse drug reaction has been reported $[8,9]$. Sex trials were trials were included in one study, 
the obvious effective rate was statistically different $[O R=11.07,95 \% \mathrm{Cl} 4.39-27.91, \mathrm{Z}=$ 5.10, $P<0.001$ ] [8], whereas ten RCTs were included in another study, the total efficiencies of the two drugs had statistical significance [ $\mathrm{RR}=12$. 96, $95 \% \mathrm{Cl}$ ( 5. 81, 28. 89), $p<$ $0.00001]$, obviously effective rates were statistically different $[\mathrm{RR}=9.11,95 \% \mathrm{Cl}$ (3.98, 20.88), $p<0.00001$, but there was no statistical difference between Ruyi Jinhuang powder and magnesium sulfate in the incidence of phlebitis and grade I skin injury in the $72 \mathrm{~h}$ of treatment $[R R=7.76$ and $0.13,95 \% \mathrm{Cl}:(0.94,64.19)$ and $(0.02,1.07), p>0.05]$ [9].

\section{Limitations of this review}

Even with these promising results, there are some limitations of the present study. First, although we were confident that our search strategy located all relevant studies, there remained a certain degree of uncertainty. Our study based on the findings of others about the heterogeneous quality of randomized trials from China. In our own experience in China, we have doubted that many methodological features attributed to randomized trials, were in fact conducted, while several explanations for this phenomenon existed, a likely explanation was the slow uptake of evidence-based medicine and clinical trials methodology in academic research centers. Second, the quality scores of the included RCTs were generally poor. Risk bias study showed that many of the studies are unclear with high risks in allocation concealment, blinding of participants and personnel, as well as outcome assessments. The unclear and high risk of bias in the included studies weakens the conclusion and well-designed randomized clinical trials are warranted to confirm the efficacy of Ruyi Jinhuang powder. While Cochrane's $P 2$ and $P 2$ tests revealed no statistical heterogeneity among these studies.

\section{Possible rationale for use of Ruyi jinhuang powder for phlebitis}

According to the TCM theory, first, the primary pathogenesis of phlebitis stagnated blood obstructing meridians and collaterals, resulting in such symptoms and signs as ischemia, thrombosis, ecchymosis and localized pain. Second, animal experiments carried out in recent years have demonstrated that phlegm-dampness firstly formed in these patients and then developed into stasis, resulting in combination of phlegm and stasis. Third, spasm and obstruction of the blood vessels will induce circulatory impairment of the affected limb, manifested by pallor, aversion to cold, distension pain, severe pain and muscle twitch. Last, damp-heat of phlebitis is manifested by redness, edema and slightly infected gangrene. Therefore, treating basic principles is to invigorate blood circulation and remove blood stasis, resolving phlegm and dredging meridians and collaterals, warming Yang and relieving spasm and cleaning up toxic heat. In conclusion, external application of Ruyi Jinhuang powder using on treating and preventing phlebitis as an important complementary therapy is based on these definite principles [26-44].

This powder can shorten the time of licking, which shown that this powder has analgesic effect $\quad(P<0.05) \quad[36,42]$. Another clinical observation of golden powder combined with external application of antibiotics on epididymitis and orchitis shown that in the clinical efficacy significantly differed $(P<0.05)$ and the scrotum in the treatment group in the signal measurement of blood levels, TCM syndrome, body temperature returned to nominal time, and the disappearance time of scrotal swelling and the disappearance time of clinical symptoms. The incidence of complications in these two groups was significantly lower than that in the control group $(P<0.05)$ and yielded no adverse reactions [56]. What's more, Jinhuang powder-polyurethane dressing, a suitable material for temporary wound coverage of traditional Chinese orthopedics, was based on the famous prescription Ruyi Jinhuang powder. Polyurethane can absorb liquid higher than nearly 15-20 times of its own weightdue to excellent suction performance. Using immersion method to make the Jinhuang powder-polyurethane dressing can have high drug-loading capacity, which can be applied to the acute wound of Guinea pig and plays a role in mitigating inflammatory responses and eliminating necrotic tissues without irritation to the skin [48].

\section{Implications for research}

As an important complementary therapy, although a substantial amount of research has investigated the chemical constituents of Ruyi jinhuang powder. However, more trials with rigorous methods of design, measurement and evaluation (DME) following the Cochrane Handbook should be applied to enhance the representativeness of the sample. Clinical trial registries should be specificallyencouraged to provide details of the protocols for treating phlebitis, placebo-controlled clinical trials are essential. Randomized controlled trials, for instance, should be strictly required in study design and reported based on the Consolidated Standards of Reporting Trials (CONSORT). 


\section{CONCLUSION}

This systematic review demonstrates positive evidence for the effectiveness of Ruyi jinhuang powder in the management of phlebitis. Nevertheless, extreme heterogeneity in the analyses remains unexplained, and the number of high-quality studies was not large enough in this systematic review; thus, the outcome of the review is not conclusive. Therefore, more highquality RCTs, with low risk of bias and adequate sample sizes, are required to demonstrate its true effects.

\section{DECLARATIONS}

\section{Conflict of Interest}

No conflict of interest associated with this work.

\section{Contribution of Authors}

The authors declare that this work was done by the authors named in this article and all liabilities pertaining to claims relating to the content of this article will be borne by them.

\section{REFERENCES}

1. Barruel GR, Cert G, Polit DF, Murfield JE, Rickard CM. Infusion phlebitis assessment measures: a systematic review. J Eval Clin Pract 2014; 20: 191-202.

2. Tagalakis V, Kahn SR, Libman M, Blostein M. The epidemiology of peripheral vein infusion thrombophlebitis: a critical review. Am J Med 2002; 113 . 146-151.

3. Tzolos E, Salawu A. Improving the frequency of visual infusion phlebitis (VIP) scoring on an oncology ward. BMJ Qual Improv Rep 2014; 3: u205455.w2364.

4. Myrianthefs $P$, Karatzas S, Baltopoulos G. Complications, thrombophlebitis rates, and intravenous catheter replacement strategies. Infection 2005; 33: 96-97, 98100.

5. Lutzow-Holm C, De Angelis P, Clausen OP. Retinoic acid provokes a regeneration-like proliferative response in murine epidermis. A bivariate DNA/bromodeoxyuridine flow cytometric study. Arch Dermatol Res 1992; 284 418-423.

6. Malach $T$, Jerassy $Z$, Rudensky $B$, Schlesinger $Y$, Broide $E$, Olsha O, Yinnon AM, Raveh $D$. Prospective surveillance of phlebitis associated with peripheral intravenous catheters. Am J Infect Control 2006; 34: 308-312.

7. Di Nisio $M$, Peinemann $F$, Porreca $E$, Rutjes $A W$. Treatment for superficial infusion thrombophlebitis of the upper extremity. Cochrane Database Syst Rev 2015; (11): CD011015.
8. Qin SW, Lan QH, Xia YH. Meta-analysis of efficacy on satisfactory golden powder to treat patients with phlebitis. The Journal of Nursing Research 2010; 24: 85-87.

9. Li GH, Jia YJ, Su S, Xia K. Evidence-Based Pharmacy Evaluation of Ruyi Jinhuang Powder for Treating Phlebitis. Chinese Pharmacology Journal 2012; 47: 1860-1862.

10. Ezzo J, Berman BM, Vickers AJ, Linde $K$. Complementary medicine and the Cochrane Collaboration. JAMA 1998; 280: 1628-1630.

11. Zhang L, Li N. Effect observation of external application of Ruyi jinghuang Powder combined with winegar in prevention of phlebitis. E-journal of Translational Medicine 2016; 3: 15-17.

12. Yang X, Ma JR, Yan XT, Cai YS, Ma WG. Curative effect observation of Ruyi Jinhuang powder combined with magnesium sulfate injection in prevention of fluorouracilinduced phlebitis of 30 patients. Hebei Journal of Traditional Chinese Medicine 2013; 35: 1184-1185.

13. Jiang YJ. Clinic observation of Ruyi Jinhuang powder packed with honey to prevent chemotherapy phlebitis of 30 patients. Fujian Medical Journal 2010; 32: 168-170.

14. Li J. Curative effect observation of Ruyi jinhuang powder combined with sodium $\beta$-seven in preventing of blistering chemotherapy drugs-induced phlebitis. Chinese Journal of Misdiagnostics 2012;12: 1080-1082.

15. Cheng HJ, Qi ZP, Wang YL. Clinical observation and nursing of topical treatment of Ruyi golden powder in the treatment of 5-fu-induced phlebitis. Chinese Journal of Misdiagnosis 2012; 12:1309-1310.

16. Zhou MR. Topical treatment of Ruyi golden powder in the treatment of chemotherapy phlebitis of 24 patients. Herald of Medicine 2013; 32:75-76.

17. Sun HX, Li HX. Therapeutic effects observation of Ruyi Jinhuang powder paste used as external treatment of chemotherapeutic phlebitis. Chinese Journal of Modern Nursing 2015;21: 4039-4040.

18. Yu ZP, Wu XQ, Liu JH. Observation in effect of wet compress with traditional Chinese medicine in treatment of chemotherapeutic phlebitis caused by indwelling needle. Chinese Journal of Practical Nursing 2010; 26: 40-43.

19. You MN, Ding Y, Shang J. Comparison of treatment effect of hirudoid and ruyijinhuang powder for phlebitis caused by chemotherapy. Chinese Nursing Management 2010; 10: 62-63.

20. Wu H. Nursing experience in the treatment of chemotherapy-induced phlebitis. Journal of Traditional Chinese Medicine 2008; 14: 64- 64.

21. Liu BR. Curative effect observation of topical treatment of Ruyi Jinhuang powder mixed with honey in the treatment of PICC-induced mechanical phlebitis. International Journal of Nursing 2011; 30:152-153.

22. Liang YF. A contrastive study of Ru-yi-jin-huang-san and $50 \%$ magnesium sulfate in treating of chemotherapeutic phlebitis. Chinese and Foreign Medical Research 2012; 10: 20-21.

Trop J Pharm Res, March 2019; 18(3):665 
23. Meng QH. Clinic nursing observation of Ruyi golden powder mixed with dense tea water in treatment of chemotherapy-induced phlebitis nursing. Journal of Qiqihar University of Medicine 2011; 32: 2522-2523.

24. Kuang CX, Shen FL, Zhou XM. Therapeutic effect observation of the external application of Ruyi Jinhuang Powder in the treatment of chemotherapy non exosmosis phlebitis, China Practical Medicine 2013;8: 174-175.

25. Wang $Y$, Ge FJ, Jin $Y Y$, Mao L. Clinical nursing observation of hydropathic compress of Ruyi golden powder in prevention of phlebitis caused by chemotherapy drugs, Asia Pacific Traditional Medicine 2014;10: 116-118.

26. He WG. External application of Ruyi golden powder in the prevention of phlebitis caused by chemotherapy drugs of 39 cases. China's Naturopathy 2012; 20: 15-16.

27. Wei HA. Curative effect observation of external application of Ruyi jinhuang powder in preventing of phlebitis caused by chemotherapy drug. Chinese Journal of Coal Industry Medicine 2009; 12: 1896-1897.

28. Liu $L H$, Yin $X Y$, Wang AZ. Effect observation of external application of Ruyi Jinhuang powder in the treatment of PICC-induced mechanic phlebitis. Guangming Journal of Chinese Medicine 2015; 30: 2345-2346.

29. Li XM, Li YS. Effect observation of Ruyi Golden Powder in treatment of phlebitis. Guide of China Medicine 2013; 20: 278-279.

30. Gao YQ, Li L. Curative effect observation of Ruyi Jinhuang powder combined with infrared irradiation in prevention of PICC-induced mechanical phlebitis. Nursing and Rehabilitation Journal 2012;11: 369-310.

31. Liu Y,Yang LY. Therapeutic effect observation of the external application of Ruyi Jinhuang Powder in the treatment of PICC-induced mechanical phlebitis. Journal of North China Coal Medical University 2009; 11: 671673.

32. Duan PP, Mei SJ, Zhang YY. The Prevention and Therapeutic Effect of Externally Applied Jinhuang Powder on PICC-induced Mechanic Phlebitis. Journal of Nursing (China) 2008; 15: 71-73.

33. Zhu LR, Li JZ, Wang XY. Observation on curative effects of Ruyi Jinhuang San mixed tea on phlebitis after PICC catheterization in elderly patients. Chinese Journal Modern Nurse 2008;14: 1463-1465.

34. Li JH, Fan AF, Zhong CC. Curative effect observation of topical treatment of Ruyi Jinhuang powder in treatment of PICC-mechanical phlebitis. The Journal of Qiqihar Medical College 2007; 28: 1245-1246.

35. Zhu AL, Chen CY, Wu J. Curative effect observation of topical treatment of Ruyi Jinhuang powder in treatment of PICC-mechanical phlebitis. Chinese Journal of Misdiagnostics 2009; 9: 2330-2331.

36. Peng W, Wang HD. The efficacy of wet compress with Jinhuang San for phlebitis after chemotherapy. Chinese Journal of Medicinal Guide 2012; 14: 135-137.

37. Shi $X Y$, Jia $H$, Fan $A F$, Gong $H Q$. Effect of Ru Yi Jin Huang powder on phlebitis during the intermittent period of PICC treatment, Journal of Hainan Medical University 2011; 17: 312-314.

38. Yao GM. Curative effect observation of Ruyi Jinhuang Powder mixed with white vinegar for the treatment of phlebitis. Medical Information 2015; 28: 269-270.

39. Huang HM. Liu Y. Effects of treatment of phlebitis by using Ruyijinhuang Powder and honey. Nanfang Journal of Nursing 2004; 11: 44-46.

40. $\mathrm{Yu} \mathrm{H}$. Curative nursing observation of topical treatment of Ruyi jinhuang powder in treatment of drug induced phlebitis. Clinical Journal of Chinese Medicine 2016; 8: 124-125

41. Wu X. Efficacy observation of Ruyi jinhuang powder mixed with Honey in treating of Kabiven injectioninduced phlebitis. World Latest Medicine Information 2015; 15:149-150.

42. Yan HL, Xu KY. Curative effect observation of topical treatment of Ruyi golden powder mixed with ethanol in the treatment of phlebitis. Today Nurse 2015; 11: 116117.

43. Liu XL, Xi H, Wang HT. Therapeutic effect observation of the external application of Ruyi Jinhuang powder mixed with green tea in treating infusion phlebitis. Public Medical Forum Magazine 2014; 18: 4840-4842.

44. Zhang LH, Dong JY, Zhang JH, Fu L. Observation on the therapeutic effects of treating phlebitis by Ruyijinhuang powder mixed with dense tea. Acta Academiae Medicinae Cpapf 2007; 16: 133-135.

45. Liu XN, Zhang YN, Quan XN. Curative effect observation of topical treatment of Ruyi Jinhuang powder mixed with honey in the treatment of amiodarone-induced phlebitis, Shaanxi Journal of Traditional Chinese Medicine 2012; 33: $1638-1640$

46. Zhang $X H$, Zhao $X Y$. Curative effect observation of topical treatment of Ruyi golden powder in treatment of amiodarone-induced phlebitis. World Latest Medicine Information 2015; 15: 165-167.

47. Dai WD. Study on external application of Ruyi jinhuang powder mixed with dense tea water in the early intervention of amiodarone-induced phlebitis, The Chinese and foreign health abstract 2013; 42: 83-84

48. Huo LR, Chang XM. Clinic observation of TDP lamp irradiation combined with Ruyi Jinhuang powder in treating phlebitis caused by amiodarone. Journal of Huaihai Medicine 2013; 31: 267-268.

49. Chen ZF, Wu J, Qian HJ, Chen LH. Comparison of effective outcome of application of Rnyi golden powder and magnesium sulfate in the treatment of phlebitis caused by amiodarrone. Chinese Journal of Practice Nursing 2013; 33: 21-24

50. Fu XR. Therapeutic effect observation of the external application of Ruyi Jinhuang Powder in treating phlebitis caused by sodium $\beta$-seven. Clinical Journal of Chinese Medicine 2016; 8: 55-56.

51. Li FL, Liu Y, Chen LQ. Ruyi Jinhuang powder wet compress plus microwave physiotherapy treatment of fat emulsion induced phlebitis curative effect observation.

Trop J Pharm Res, March 2019; 18(3):666 
Journal of Hunan University of Chinese Medicine 2013; 33: 36-37.

52. Yang WJ. Ruyi. Efficacy comparison of topical treatment of golden powder combined with magnesium sulfate in the treatment of mannitol-induced phlebitis. Journal of Psychological Doctors 2012; 10: 292-293.

53. Tang G, Meng JH. Curative effect observation of topical treatment of Ruyi jinhuang powder combined with glycerinum in the treatment of Gadopentetate dimeglumine injection-induced phlebitis. Guangming Journal of Chinese Medicine 2012; 27: 260-262.

54. He YH, Zhao HM, Han X. Clinic observation of topical treatment of Ruyi Jinhuang Powder mixed with vinegar for phlebitis caused by allitride injection. Today Nurse 2009; 2: 60-63.

55. Li RB, Wang S, Hu XN. Effect of dermlin dressing applying in elderly patients with superficial phlebitis. Chinese Journal of Nosocomiology 2010; 20: 795-797.

56. Mei R. Comparison of effectiveness between dermlin and the powder of Ruyij inhuang on superficial phlebites caused by long-term continuous intravenous injection treatment. Journal of Nanchang University (Medical Science) 2010; 50: 78-79.

57. Zhang SZ, Wang Y. A study of prevention of the occurrence of superficial phlebitis with externally applied Ruyi Jinhuang powder. Medical Information 2013; 26 : 28-29.

58. Zhou FF, Fan ZR, Xiao ZA. Clinical observation of topical treatment of Ruyi golden powder in prevention of venous indwelling needle-induced phlebitis caused by continuous drip 5-fu. Journal of Qilu Nursing 2012; 18: 28-29.
59. Yu XY, Tan HY, Du XY. Clinical observation of topical treatment of Ruyi golden powder in the treatment of indwelling needle-induced phlebitis. Chinese and Foreigh Medical Research 2010; 8: 57-59.

60. Li XY. Clinical effects of Ruyi golden powder in patients with superficial venous indwelling needle and phlebitis. Nursing Journal of Chinese People's Liberation Army 2012; 29: 71-73.

61. Xu JX, Hong QX, Jiang YS, Le YH, Hong Guo. Efficacy observation $50 \%$ alcohol harmonic ruyi jinhuang powder combined with lamp local irradiation for the treatment of indwelling needle-phlebitis. Medical Information 2011; 24: 3792-3793.

62. Song LL, Zhang QM, Shi JY. Curative effect observation of topical treatment of Ruyi Jinhuang powder mixed with Sesame oil in treatment of venous indwelling needleinduced phlebitis. Chinese Journal of Geriatric Care 2014; 12: 113-115.

63. Liu ZX, Zhou ZF. Curative effect observation of topical treatment of Ruyi jinhuang powder mixed with honey on phlebitis caused by intravenous indwelling needle. Guiding Journal of Traditional Chinese Medicine and Pharmacy 2012; 18: 126-127.

64. LA Gorski. Infusion Nursing Standards of Practice. Journal of Infusion Nursing the Official Publication of the infusion nurses society 2007; 30: 151.

65. Higgins JP, Altman DG, GotzscheP $C$, Juni $P$, Moher $D$, Oxman AD, Savovic J, Schulz KF, Weeks L, Sterne JA. The Cochrane Collaboration's tool for assessing risk of bias in randomised trials. BMJ 2011; 343: d592. 